\title{
In situ statistical measurement of local morphology in carbon-epoxy composites using synchrotron X-ray computed tomography
}

\author{
Sebastian Rosini ${ }^{a,},{ }^{*}$ Mark N. Mavrogordato ${ }^{a}$, Olga Egorova ${ }^{b}$, Emily S. Matthews ${ }^{b}$, \\ Samuel E. Jackson ${ }^{b}$, S. Mark Spearing ${ }^{a}$, Ian Sinclair ${ }^{a}$ \\ ${ }^{a}$ Department of Mechanical Engineering, University of Southampton, Southampton, SO17 1BJ, United Kingdom \\ ${ }^{b}$ Department of Statistics and Mathematics Sciences, University of Southampton, Southampton, SO17 1BJ, United Kingdom
}

\begin{abstract}
Models are still deficient in accurately reproducing the mechanisms that trigger tensile failure in unidirectional composites, highlighting a lack of direct experimental evidence. In this study, emphasis is given to the identification of connections between local fibre misorientation, packing and Weibull strength distribution in causing tensile failure. Synchrotron Radiation Computed Tomography (SRCT) and automated image processing techniques are adopted to segment individual fibres from loaded carbon fibre coupons. Subtle indications in the misorientation of local damaged sites are assessed in novel statistical detail for systematic differentiation from non-damaged sites. It is observed that the morphology of the surrounding environment of damaged sites statistically differs from that of non-damaged sites, even though locally damaged sites (containing single or coupled breaks) do not exhibit a peculiar fibre packing arrangement. For adjacent coupled breaks, the statistical nature of fibre separation distances is also reported.
\end{abstract}

Keywords: Carbon fibres; tensile failure; fibre misorientation; Synchrotron Radiation Computed Tomography

${ }^{*}$ Corresponding author

Email addresses: s.rosini@soton.ac.uk (Sebastian Rosini),mnm100@soton.ac.uk (Mark N. Mavrogordato), I.Sinclair@soton.ac.uk (lan Sinclair), S.M.Spearing@soton.ac.uk (S. Mark Spearing), O.Egorova@soton.ac.uk (Olga Egorova), E.S.Matthews@soton.ac.uk (Emily S. Matthews), sej1a18@soton.ac.uk (Samuel E. Jackson)

\section{Introduction}

Polymer matrix composites represent a diverse class of materials widely used in transport applications for their high specific properties and adaptability. Models predicting the failure performance of such materials for engineering purposes are not well established however, leading to over-engineered and hence heavier, larger and more expensive components [1]. This has been attributed to the complex micromechanical interactions within and between the matrix, reinforcing fibres and multi-orientation laminar layups, engendering multiple potential damage modes within any given component, e.g. matrix cracking, fibre-matrix debonding, microbuckling, delamination and fibre failure [2]. Attempts to predict the failure of multidirectional composites under different loading conditions have been extensively tested within the World Wide Failure Exercises I, II, III [3-5] in which numerous modelling strategies have been 
assessed in detail and compared to reference experimental data sets. The results showed a significant spread in the predictions, highlighting the need for many model input properties (often difficult to measure), as well as challenging experimental validation [1]; a simpler approach might be preferred to capture correctly the fundamental mechanisms.

Within a multi-orientation laminate, it can be expected that the ultimate tensile strength will be limited by essentially simple tensile separation of the $0^{\circ}$ plies (i.e. plies aligned with the primary tensile load) $[2,6]$. This process has been analysed historically, with various modelling strategies reported in the literature based on essentially the same fundamental assumed mechanisms [7,8]:

- Within a given $0^{\circ}$ ply, as the longitudinal stress increases, failure of the weakest fibres occurs (according to a statistical strength distribution [9]), generating stress concentration on immediately neighbouring intact fibres.

- As loads increase, fibres neighbouring breaks are more likely to fail than those in the surrounding microstructure, leading to local clustering of breaks.

- From this, a cascade effect is eventually achieved, where a critical cluster of broken fibres grows in an unstable, self-sustaining manner.

In this context, the local role of the matrix is to load in shear and transfer the stress back onto the broken fibre, as well as to transfer stress onto the fibres surrounding the broken one, generating stress concentration on these for a certain distance from the break position along their length. In the literature, this is indicated as the ineffective length [10] with experimental measurements for carbon fibre reinforced polymers (CFRPs) provided by Scott et al. [11], as an example.

Although the process of fibre break accumulation can be seen as conceptually simple, various issues arise in the corresponding predictive models, for example, the experimental determination of appropriate Weibull distribution parameters and the approximations used to model the fibre and matrix mechanical behaviour. The latter commonly entails the assumption of the fibre and matrix being linear elastic, and the fibre-matrix interface being intact, in the simplest case. In reality, the matrix shear yields or cracks and fibre-matrix interface debonding is often observed in composites with poor adhesion [1]. Commonly made assumptions in strength models are extensively described by Swolfs et al. in [6], including alternative representations of fibre packing, stress redistribution around single and multiple fibre breaks, and the potential influence of dynamic stress concentrations. Other assumptions that have received little attention in the literature until now may also be identified in stress variation within the cross-section of intact fibres surrounding breaks, the incidence of planarity (or nonplanarity) of groups of breaks (some models assume full planarity of groups of breaks [12]). Assumptions vary regarding the onset of unstable break clustering causing final failure (whether it should be a fracture mechanics approach rather than a stress-based one), whilst Swolfs et al. further identify the absence of local fibre microstructure in contemporary state-of-the-art models.

Models that predict the accumulation of interacting fibre breaks can be retrieved in the literature and to be cited are the hierarchical scaling law [13]; the spring element models [1416] (inspired by the Zhou and Curtin's lattice Green's function model [17]) and following evolutions of this model that include dynamic effects on the break formation, such as $[18,19]$; the direct numerical simulation method that used a fibre-bundle model similar to the 
abovementioned spring element model $[1,20,21]$; the multiscale $\mathrm{FE}^{2}$ model that uses the $\mathrm{FE}$ analysis of composites at the microscale and includes time-dependent visco-elastic effects and homogenisation methods [22-26]. These models have been tested against experimental results and in some cases a good agreement with the single fibre break predictions is reached (as reported in $[2,18])$, while the clustering prediction is still inaccurate, suggesting a higher level of understanding of the features governing the longitudinal tensile failure of composites is needed.

Despite fibre strength distributions clearly playing a key role in strength models, the significance of the assumptions outlined above is not clear. Hence, in this study we have performed a detailed analysis of local fibre microstructures, with a view to providing statistically significant indications of potential influence on the formation of single fibre breaks, and groups of closely interacting breaks.

Focus is given to planar coupled breaks: since they are available in a good amount in the collected data to draw statistical conclusions, and since, according to the majority of models, should play a crucial role in increasing the stress concentration around a break site and the recovery length if compared to diffuse clusters of breaks [27], and as predictive models are still deficient in accurately tracking their formation and evolution, as noted in [28] where the results of an SRCT experimental campaign were compared to the modelling predictions. In this respect, in situ X-ray SRCT has emerged in the last decade as a unique tool to quantify microstructure and damage in composite materials, and follow the chronology of damage progression, providing the modelling community with novel experimental validation opportunities at a micromechanical level.

In studying the morphology of continuous fibre composites via computed tomography, previous studies have assessed the presence of misorientation in carbon fibre reinforced polymers (CFRPs). A multiple field image analysis method was adopted in [29], well suited for coupons with pronounced waviness, while a method applicable to small volumes with limited amount of fibres is adopted in [30]. However, no results are presented on in situ following the damage accumulation. Emerson et al. ([31]), have reached a fibre-by-fibre detection level, characterising orientation in glass and carbon fibre composites from relatively low contrast images, and presented quantitative results on the axial compression of composites in [32]. Where Emerson's algorithm comprises a training process to generate a dictionary that reduces the degree of false centre detection, in this study a simple Otsu's threshold ([33]) has been shown to track fibres accurately. Although in both cases about 7 pixels covered a fibre diameter, the approach used in the present work can be justified by the better quality of the scans obtained through SRCT rather than laboratory-CT (beam is brighter, monochromatic, more coherent and parallel providing less noisy images, with a higher level of phase contrast and absence of beam hardening artefacts [34]). However, where Emerson's algorithm is proven to accurately track fibres over lengths of millimetres and focus on larger lengths to analyse the fibre orientations, focus is here given to shorter lengths (below $100 \mu \mathrm{m}$ ) and to the centre detection accuracy within the cross-section of a fibre, checking at a sub-micron level with a meticulous error trapping approach.

In this study we exploit previously presented damage monitoring methods (see $[11,35,36]$ ), and, to the authors knowledge, provide the first automated approach to quantify at the individual fibre level the local microstructure at single and groups of adjacent fibre breaks locations in statistically significant numbers. By combining for the first time SRCT, automated image processing techniques and statistical tools, the study represents a first step towards a full 
microstructural analysis of composite coupons in situ tested using SRCT, that can inform on the geometry of large groups of interacting fibre breaks, believed to drive the longitudinal tensile failure mechanisms in composites. The damage progression is not only followed in terms of fibre breaks from low to high applied strains but also the morphology of all the fibre break sites is captured prior to failure. The results of the method adopted for the analysis in this study show that it is possible to capture subtle indications in the fibre misorientation and spacing distributions in the single fibre break and intact sites, as these are features available in sufficient amount in the analysed material. Nevertheless, where possible results will be also presented on coupled planar breaks, that represent the planar features still observable in a small-to-average amount to draw statistical conclusions. The final aim will be to perform a test campaign that captures a higher number of large n-plets, believed to drive the longitudinal tensile failure mechanisms in composites and statistically observe their morphology comparing it to the morphology presented in this study of non-damaged, single and coupled fibre break sites. This could benefit future experimental analyses, e.g. in recognising a priori areas of peculiar morphologies that could be in situ followed from low to high strain for group of interacting fibre break formation that could lead to the failure and inform the modelling community on uncharacteristic morphologies (e.g. regions of fibre waviness, entanglement or resin rich regions) that should be included in the models to fully capture the mechanisms leading to longitudinal final failure of composites.

\section{Materials and experimental procedure}

\subsection{Materials}

$\left(90_{2} / O_{2}\right)_{s}$ laminates were produced from Mitsubishi Chemical Co. carbon fibre pre-preg to the manufacturer's specifications; the nominal fibre diameter was $5.4 \mu \mathrm{m}$ and the tensile modulus $289 \mathrm{GPa}$. The tensile strength follows a Weibull distribution, with a shape factor of 5.47 and a scale factor of $11.29 \mathrm{GPa}$, at a gauge length of $5 \mathrm{~mm}$. The average strength calculated by Weibull distribution is of $8.07 \mathrm{GPa}$. This matches the experimental average strength of $7.76 \mathrm{GPa} .1 \mathrm{~mm}$ thick double edge notched tensile test coupons were realised via waterjet cutting, as indicated in Wright et al. [35], see Fig. 1. Aluminium tabs were carefully glued to ensure alignment of the sample during tensile loading in the electro-mechanical rig. The average ultimate tensile stress $\left(\sigma_{f}\right)$ measured across the notched section was $3.47 \mathrm{GPa}$. Two coupons were investigated in this study (identified as coupon 1 and coupon 2), to assess levels of reproducibility. About 8000 fibres were present within the notched region, with a gauge length of $\sim 1.3 \mathrm{~mm}$ being imaged in the field of view of the scans.

\subsection{Experimental procedure}

In situ tensile tests were performed at the European Synchrotron Radiation Facility (ESRF) in Grenoble, France (ID19 microtomography beamline). A modified DEBEN CT5000 tensile stage (Fig. 1 (a)) was used to carry out the tests, as reported previously [37]. The central notched region of the coupons (see Fig. 1(b)) was initially scanned at $\sim 50 \%$ and $~ 80 \%$ of the nominal average ultimate tensile strength (UTS) for the coupon geometry, with at least ten more scans being collected between $\sim 80 \%$ UTS and final tensile failure of the coupon. Tests were carried out at a displacement rate of $0.2 \mathrm{~mm} / \mathrm{min}$, being halted briefly for each scan (individual scan duration was $\sim 2.5 \mathrm{~min}$ ), the tensile force was backed off by $\sim 10 \%$ during the scan, for mechanical stability and to optimise image quality for subsequent quantitative analysis. 


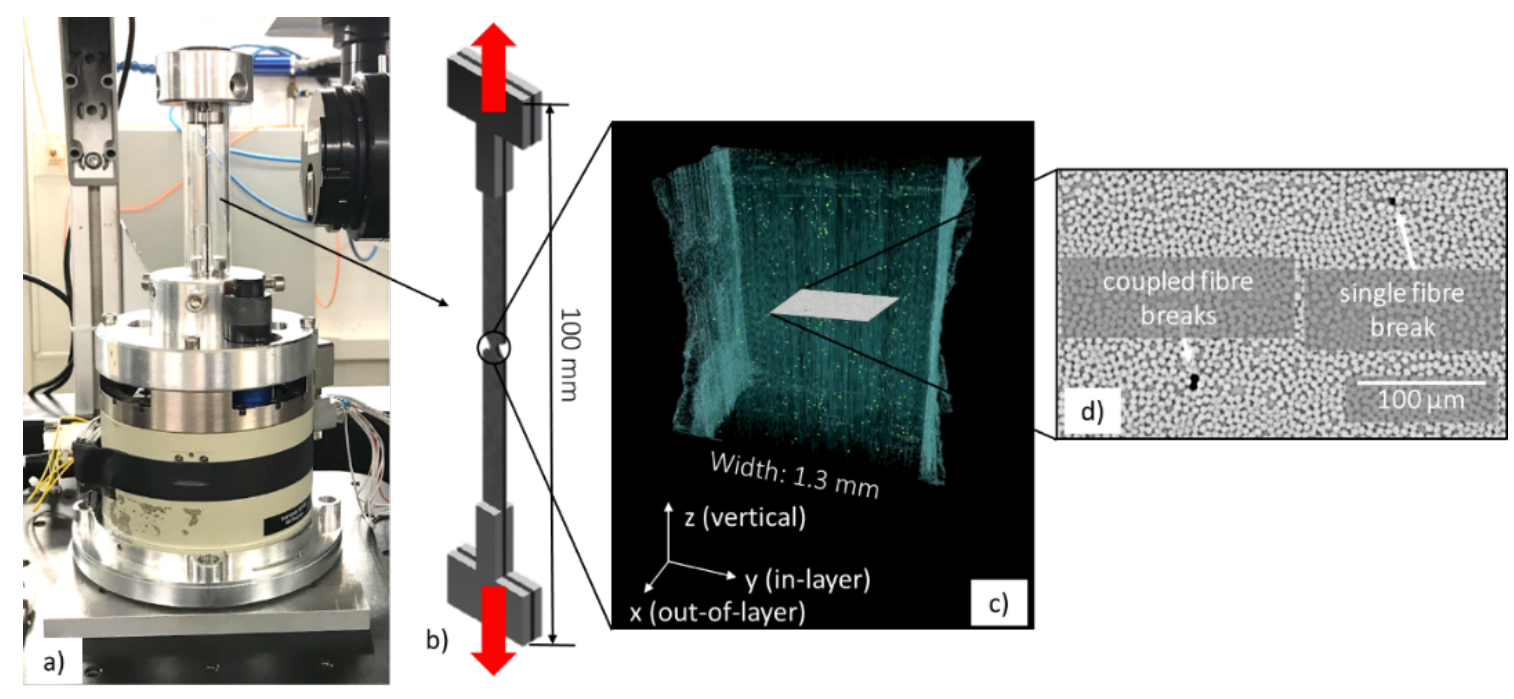

Fig. 1 (a) Illustration of the modified DEBEN CT5000 loading rig on the experimentation stage at ESRF ID19, (b) coupon geometry, (c) a 3D representation of the imaged volumes for all the scans and (d) a top view of the features detected within the volume (single fibre breaks or groups of co-planar fibre breaks. In this case a duplet is highlighted).

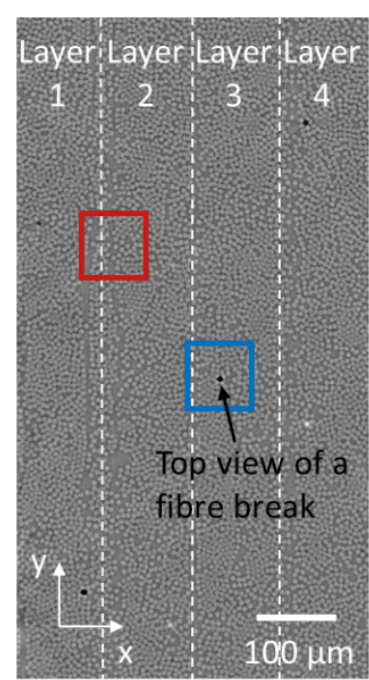

$\square$ Top view of an intact site $\square$ Top view of a singlet site

a)

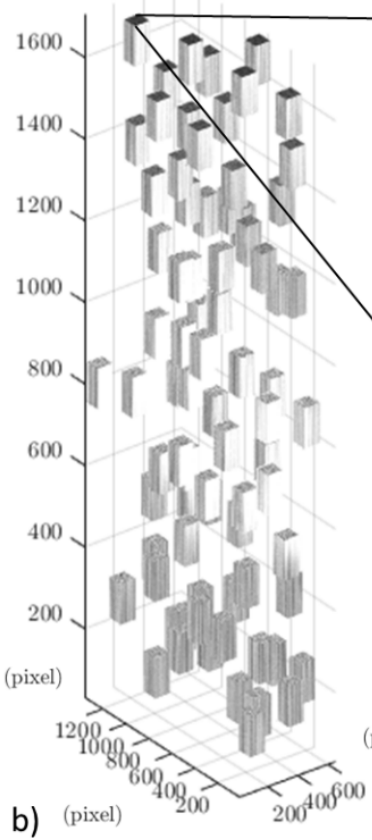

b) (pixel)

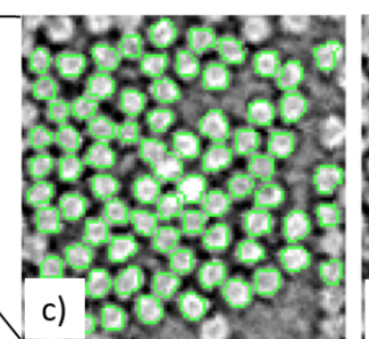

c) $26 \times \mathrm{ar}:$

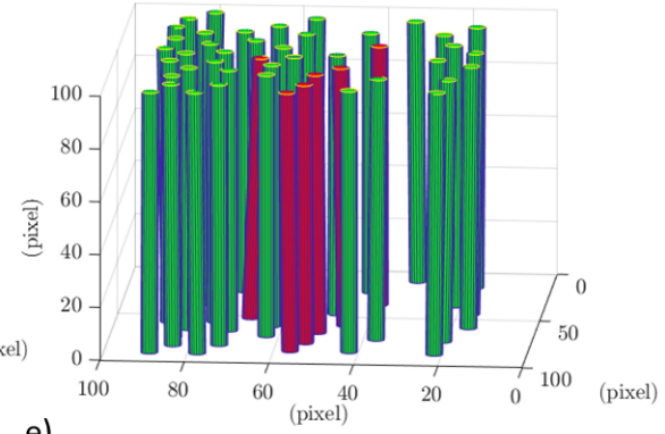

e)

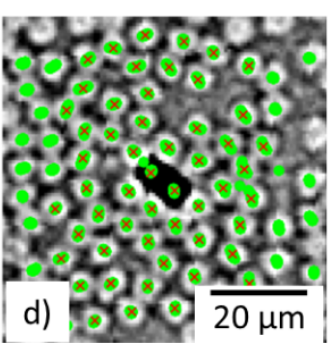

$20 \mu \mathrm{m}$

Fig. 2 Misorientation data extraction methodology: (a) Single top view slice of $0^{\circ}$ ply at high strains (immediately prior to macroscopic failure of the coupon). Locations for sub-volume extraction are centred appropriately between single, coupled fibre break sites or random nondamaged sites. (b) 3D sub-volumes extracted from the scanned volume. (c) Fibre tracking: on a single top view slice of a sub-volume, an image enhancement approach is adopted to segment the fibres. After this, an algorithm recognises and catalogues one by one all the fibres centres in a sub-volume, tracing continuity between all slices, which effectively removes occasional false detection on a single slice or occasional small group of slices. (d) in black at the centre of the image, two co-planar breaks - red crosses indicate candidate fibres location used in the tracing 
analysis. (e) Fibres in a sub-volume are visualised: more misaligned fibres (misorientation greater than $1.5^{\circ}$ with respect to loading direction) are here represented in red; in green, fibres having a lower degree of misorientation.

Voxel resolution provided by the optics in this study was $\sim 0.65 \mu \mathrm{m}$ (sufficient to observe individual fibre breaks) with a field of view of $\sim 1.3 \mathrm{~mm}$, see Fig. 1(c) in which a 3D representation of the imaged volume for all the scans is shown. Exposure time was $50 \mathrm{~ms}$ and the number of projections 2996. A multilayer monochromator was used $\left(\Delta \mathrm{E} / \mathrm{E} \sim 10^{-2}\right)$ at an energy of $19.5 \mathrm{kV}$ and sample-to-detector propagation distance of $50 \mathrm{~mm}$ (near-field Fresnel/edge enhancement regime).

An example scan immediately prior to failure ( 99\% of the UTS) is shown in Fig. 1(c), along with the reference axes of this study: with $x$ and $y$ respectively indicating the out-of-layer and inlayer transverse directions, with $\mathrm{z}$ the loading direction. Crack opening due to fibre breaks was well in excess of the voxel size for all the collected volumes of scans, with breaks being readily detected via automated approaches, see highlighted top view of one single (singlet) and two coupled fibre breaks (duplet) in Fig. 1 (d).

\section{Image analysis}

Automated image processing was carried out in Image $\mathrm{J}^{T M}$ and MATLAB ${ }^{\circledR}$. Fig. 2 illustrates the approach adopted to observe fibre misorientation at individual damaged sites, as explained below.

\subsection{Individual fibre tracking and sub-volumes extraction}

An Image $\mathrm{J}^{T M}$ ([38]) macro is used to load the last collected volume of a given set of sample scans, just prior to failure, which thresholds the fibre break sites and save information on their morphology (spatial coordinates, dimensions, volume, orientation, etc.). Based on this, a MATLAB ${ }^{\circledR}$ script catalogues the breaks into singlets and planar duplets (see Section $\S 4$ ). Given the number of planar multiplets observed ( $\sim 3 \%$ to $5 \%$ of all break sites) and their complex geometry, a final visual inspection of these sites allows the user to visually confirm the automated measurement of the number of fibres associated with each site. A second MATLAB ${ }^{\circledR}$ script that uses the Image Processing Toolbox [39], takes the last collected scan volume prior to failure and extracts for each fibre break site a sub-volume centred on the break that includes some 30 to 50 fibres surrounding the broken one, $\sim 0.65 \mu \mathrm{m}$ long, as shown in the blue box in Fig. 2(a). The script also extracts sub-volumes at coupled fibre break locations and a randomly selected set of intact locations, separate from break locations and from each other to be reasonably uniformly distributed through the whole volume (see Fig. 2 (a),(b)). Measurements at the intact locations are used to characterise as the 'background' microstructure of the material. Thus, sub-volumes are divided into: sub-volumes centred on a single fibre break (hereafter indicated as 'singlet sites'), damaged sub-volumes centred on two planar adjacent breaks ('duplet sites') and sub-volumes containing non-broken fibres ('intact sites').

Within a sub-volume, the 'imadjust' function is used to enhances the contrast of the images containing the top views of the fibres prior filtering with the 'imgaussfilt' function: a 2-D Gaussian filter that reduces the contrast but at the same time lowers noise within the resin regions, which is the principal cause of false fibre detection. The fibres are then morphologically opened with the 'imopen' function: an erosion followed by a dilation, using the same disk-shaped structuring 
element to facilitate the fibre thresholding. After this, the 'imbinarize' function is adopted to threshold the fibres by means of a globally determined threshold value obtained with Otsu's method [33]. Finally, the 'regionprops' function provides the centre of each fibre detected in the 2-D slice. Fibres are segmented on every tenth slice (perpendicular to the fibre direction, as indicated in Fig. 2 (c)), for a total of ten steps spaced $6.5 \mu \mathrm{m}$ from each other. The 'rangesearch' function is adopted that works as follow: for each fibre centre in slice $i$, its correspondent is found in slice $i+1$ that has a difference in coordinates of less than a certain distance (initial tests showed 2 pixels, equivalent to $1.3 \mu \mathrm{m}$ was a good compromise for the analysed material). Two centres of a same fibre detected at an axial distance of $6.5 \mu \mathrm{m}$ that have a transversal distance larger than $1.3 \mu \mathrm{m}$ are rejected, i.e. an angle with the vertical loading direction of more than $12^{\circ}$ is not accepted: fibres in this study have been observed to have a degree of misorientation that can reach up to $8^{\circ}$ with respect to the vertical direction of the CT scans.

If a centre is missed on one slice, the script keeps the same coordinates of the centre and moves to the next slice. The 'regionprops' function provides the coordinates of the centre of a fibre with four decimal digits. If these digits do not change for at least half the number of slices investigated, then this fibre candidate is rejected. The results of the script have been visually checked for 40 sub-volumes containing an average of 55 fibres each on a total of about 2200 analysed fibres, showing the following errors: 7 false negatives and 7 false positives, representing an error of $0.006 \%$ in fibre detection.

Detected fibre centres are connected by linear interpolation, and fibres recognised and catalogued individually. Sub-volumes are slightly larger than needed to ensure fibres in the area of interest are well tracked as shown in Fig. 2 (d), where the fibres assessed in relation to the central duplet (in black at the centre of the image) are indicated with a red cross. Fig. 2(e) illustrates fibres from a typical single sub-volume, where fibres with a misorientation greater than $1.5^{\circ}$ (with respect to the coupon loading direction) have been distinguished in red from their more closely aligned neighbours (green).

\subsubsection{Estimation of fibre orientation errors}

Sub-pixel accuracy in fibre tracking is achieved by fitting to centroids measured on multiple slices, following the fibre trajectory and, ameliorating local errors/noise in the centre detection on any one slice of within a sub-volume. Linear regression is adopted to get the best fit of the fibre centres. The linear regression is separately applied to the $x$ coordinates of the fibres and then to the $y$ coordinates obtaining a smooth straight shape for each fibre. Mean $R^{2}$ values evaluated for a sample of 2500 fibres extracted from coupon 1 were of 0.65 and 0.71 , respectively in $x$ and $y$ directions, with a standard deviation of 0.30 and 0.31 . However, the correction given by the linear regression approach was always well below a pixel size. A visual approach was adopted for which fibres have been checked in the scanned volume and within a length of about $100 \mu \mathrm{m}$ (well in excess of the $65 \mu \mathrm{m}$ of the sub-volume length): fibres appeared as straight and did not show any signs of waviness. This was checked on a large number of sites ( 40).

As a further error check step, the sub-volume length is changed from 65 to 52 and then $78 \mu \mathrm{m}$. If fibres are correctly detected, their misorientation should be identical if detection is done on a smaller or larger length, i.e. detection performed on a lower or higher number of slices should lead to the same fibre misorientation. An example is shown in Fig. 3 (a): varying lengths of fibre are presented for single broken fibre sites. Over 100 sites with 40 by $40 \mu \mathrm{m}$ cross-sections were 
assessed. Circular selections centred on the fibre breaks were assessed, containing an average of 7 fibres per site. Fibre misorientation was checked separately in the in-layer and out-of-layer directions, using the displacement between the two ends of the centres of the fibres at the top and at the bottom faces of the sub-volumes, i.e. considering the coordinates $\left(x_{1}, y_{1}, z_{1}\right),\left(x_{2}, y_{2}, z_{2}\right)$ and calculating for the in-layer direction:

\section{Equation 1}

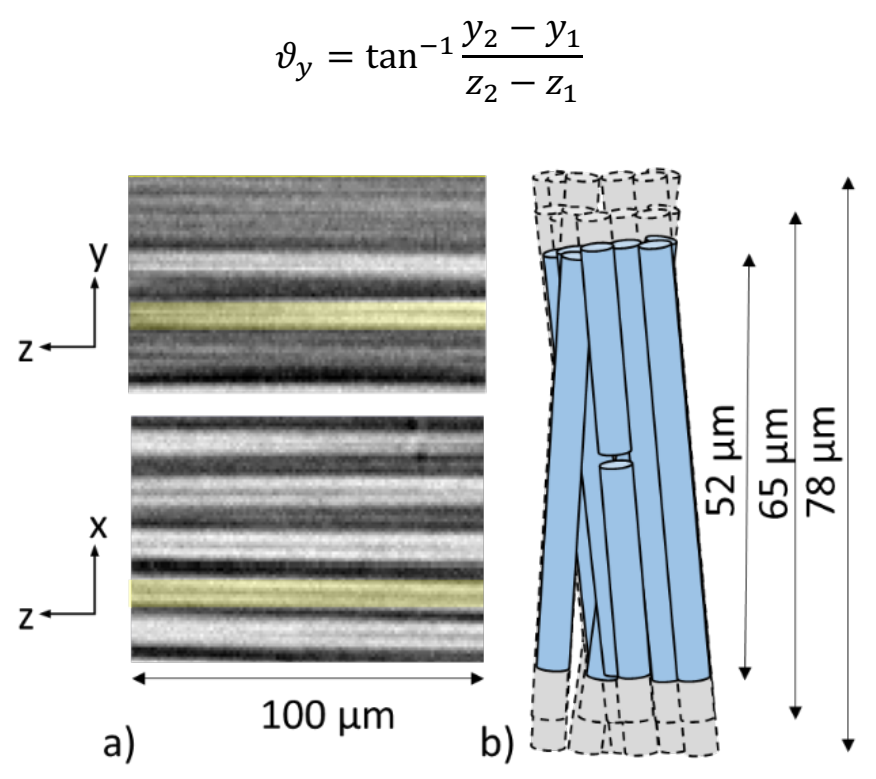

Fig. 3 The length of the same sites centred on a single broken fibres is varied from 52 to 78 $\mu \mathrm{m}$. Over 100 sites with a 60 by 60 pixel (about 40 by $40 \mu \mathrm{m}$ ) cross-section were assessed. Fibres were detected in circular selections centred on the fibre breaks, containing an average of 7 fibres per site.

where $z_{2}-z_{1}$ equals the sub-volume length. The histograms of the fibre misorientation angles in the in-layer direction for the three different-length sub-volumes centred on the same fibre breaks showed clear superposition. A two-sample Kolmogorov-Smirnov (KS) test has been used to compare the three distributions of data, being a non-parametric method to compare samples, sensitive to differences in location and shape of the samples cumulative distribution functions [40]. The distributions were confirmed to be drawn from the same statistical population (with a p-value of 1.0), i.e. more confidence can be given to the fibre centre detection, as changing the tomographic slice at which the centre is detected does not change the fibre orientation value obtained.

In this study, the number of observations played a relevant role in maximising the statistical value of the work: choosing long sub-volumes effectively reduced space available in the main volumes to extract separate sub-volumes for comparison. Hence, a length of 100 pixels (equivalent to $65 \mu \mathrm{m}$ ) was chosen, allowing a good number of sub-volumes to be extracted from the main scans, but still permitting accurate representation of local fibre orientations. 


\subsubsection{Identification of local microstructure length scales}

For the two analysed coupons, a total number of 199 singlet sites and 23 planar duplet sites were extracted from the main scanned volumes. Given the amount of volume left after the singlet and duplet sites extraction, it was possible to extract a slightly lower number of intact sites (specifically, 183) which did not overlap with any of the break sites.

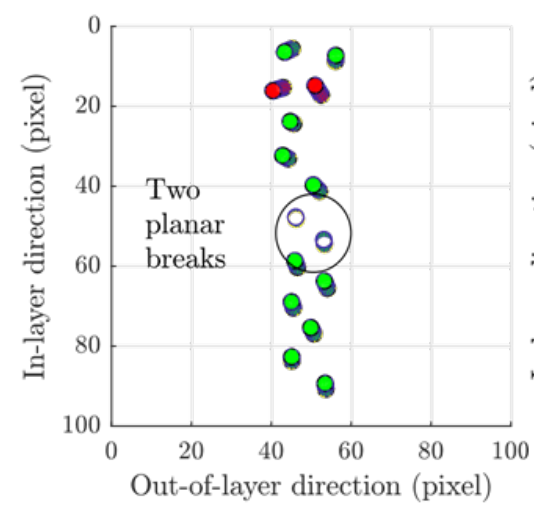

(a) Width 1: 2 fibre diameter

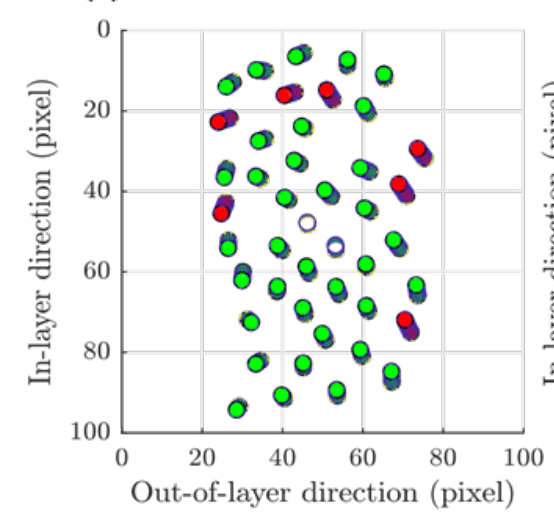

(c) Width 3: 6 fibre diameter

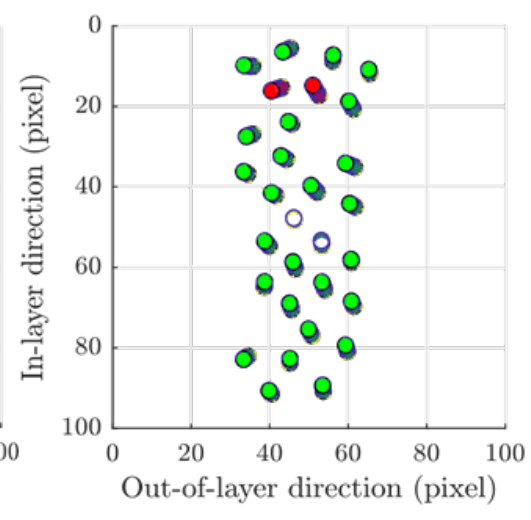

(d) Width 2: 4 fibre diameter

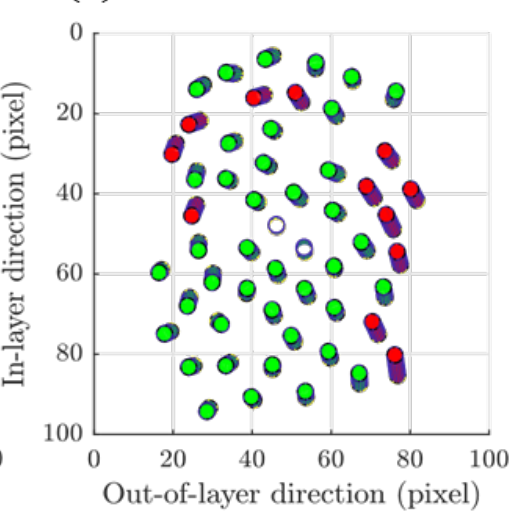

(d) Width 4: 8 fibre diameter

Fig. 4 Different microstructural sampling scales have been analysed within a sub-volume (with a different number of fibres being extracted from rectangular cross-sections having the larger dimension parallel to the in-layer direction). Width 1 is of two fibre diameters, increasing of 2 fibre diameters up to Width 4, 8 fibre diameters wide. Although all the width-steps were analysed, width 2 was chosen for the results section, being at a scale local enough to capture variability in the fibre misorientation between damaged and intact sites. Rationale of the choice is described in §4.3.1.2.

The length-scale over which patterns of fibre misorientation may or may not affect fibre break events is considered to be unknown here. For example, is one 'rogue' near-neighbour fibre sufficient to influence failure, or may disruptions or intersections at tow level lead to local changes in compliance and hence local overload of fibres. Thus, different microstructural sampling scales have been tested, presuming that too small a sampled region may not capture enough local structure to be mechanistically meaningful, but too large may statistically overwhelm the real microstructural influence with surrounding unrelated measurements. In the first instance, a rectangular cross-section was chosen for local microstructural assessment, for its simple shape and reflection of some expected difference in how in-layer and throughthickness misorientation arise. To assess length scales, different region widths in the through- 
thickness direction are assessed here, from two fibre diameters ('Width 1 ') to eight fibre diameters ('Width 4'), see Fig. 4. Although all width steps were analysed, focus was given here to Width 2. The rationale for this choice can be found in §4.3.1.2 where it is shown that this step seems to be enough at a local scale to capture variations between damaged and not-damaged sites When not specified, the following considerations will be based on data extracted from sites having this width. Misorientation angle values were recorded for: the single broken fibres and the fibres surrounding these, the planar coupled broken fibres and the fibres surrounding these, the intact fibres at the centre of the 'intact' sites and the fibres surrounding these.

\section{Results and discussion}

As noted above, image analysis was carried out for two representative coupons cut from the same CFRP panel (Coupon 1 and Coupon 2). Qualitative observations suggested some influence of fibre misorientation and coordination on damage processes: Fig. 5 for example, shows a site of particularly intense damage (2D slice views in images (a)-(c)) where 7 closely associated fibres have been segmented and coloured for clarity (Fig. 5 (d), (e)). The 3 fibres highlighted in green in Fig. 5(d) all break at 4 sites along their length (field of view $\sim 1.3 \mathrm{~mm}$ ): as a group, these 3 fibres are seen to be twisted around a number of their neighbours (in yellow), but are in themselves closely packed and retain a consistent coordination with each other. A further fibre that is highlighted in blue is initially separate from green group, but at a point of close approach with the green group, experiences breaks at these same locations. The abovementioned represent two of the four 4-plets captured in the prior-to-failure scans of the two analysed coupons. No larger multiplets were observed in the analysed scans and for this material configuration.

The influence of such specific fibre arrangements might of course be taken forward and investigated by direct microstructural finite element (FE) simulation [20]. In the first instance, we note that: (a) by eye, there is a high level of self-similarity within the microstructure, making it difficult to discern if behaviour at a site such as Fig. 5 is indeed truly distinctive from the surroundings, and (b) the statistical variability of fibre strengths may be anticipated to superimpose upon local microstructural influence, complicating apparent causality. As such we believe that break behaviour merits quantitative, statistical analysis of microstructure at damage sites against what might be considered 'background' undamaged microstructure, as outlined in $\S 3$. A hypothesis-led approach is therefore adopted. In the first instance, we outline five main comparisons here, as illustrated in Fig. 6.

Hypothesis 1: The orientation of the individual fibres that actually break is distinctive from the general, undamaged microstructure. As such the fibres that break (i.e. those at the centres of the singlet/duplet sites described in §3.1), are statistically compared to fibres within intact sites.

Hypothesis 2: The orientation of fibres that break is distinctive from their immediate environment. For example, indicating if an otherwise well aligned fibre surrounded by a region of misorientation is more likely to break. As such, the orientation characteristics of fibres that break are compared to their immediately surrounding environment.

Hypothesis 3: The environment surrounding singlet/duplet sites is distinctive from equivalently proportioned regions where breaks have not occurred. As such, the orientation characteristics of fibres within singlet/duplet sites are compared to those within intact sites. 
Hypothesis 4: The local fibre packing and the scatter in the local fibre misorientation influence the failure process. The number of fibres within a site and the standard error of their misorientation are quantified to identify a peculiar combination of the two factors.

Hypothesis 5: This focusses on the incidence of coupled fibre break duplets, testing if the nearest-neighbour fibre separation at duplet sites is distinctive from nearestneighbour distances where either a single fibre break has been recorded, or the nearestneighbour distances drawn from the general unbroken microstructure.

With the many thousands of individual fibre measurements that are readily derived from the fibre segmentation, many other tests/hypotheses are possible - four are considered here for brevity. As stated in $\S 2.2$, it is noted that measurements presented here are based on the final load step (between 98 and $99 \%$ of coupon UTS). Even at such high relative loads, the number of high multiplet sites $(n \geq 3)$ is limited: as such, only the statistics associated with duplets has been examined here, representing at least an initial stage in progressing from single fibre break to critical cluster formation.

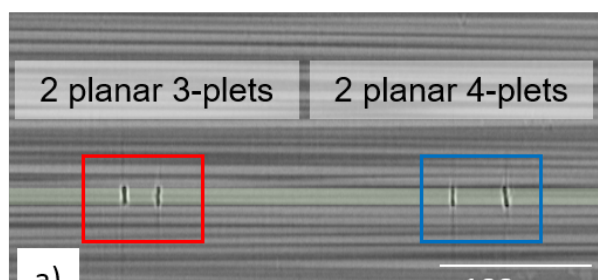

a)

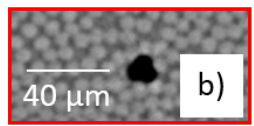
$100 \mu \mathrm{m}$
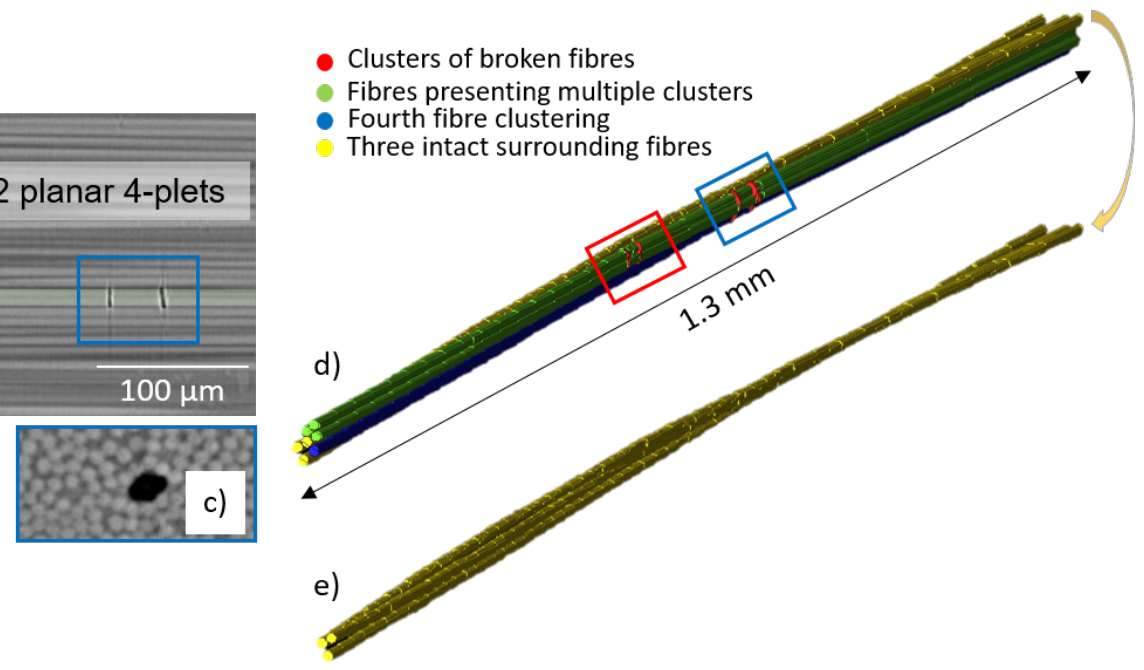

Fig. 5 Three fibres break four times along their captured length in the field of view of the scans. A fourth fibre breaks twice with them when touching them. In (a-c), the lateral and top view of the adjacent multiplets; in (d) damaged site as segmented from tomographic scans. In green the three fibres breaking four times, in blue the fibre breaking twice with the green ones, when in close proximity (e) the yellow surrounding intact fibres are shown, with their distinctive twisting. 


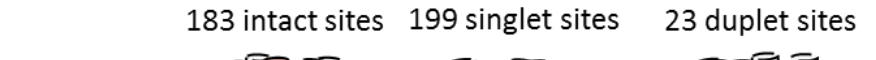

Hypothesis 1
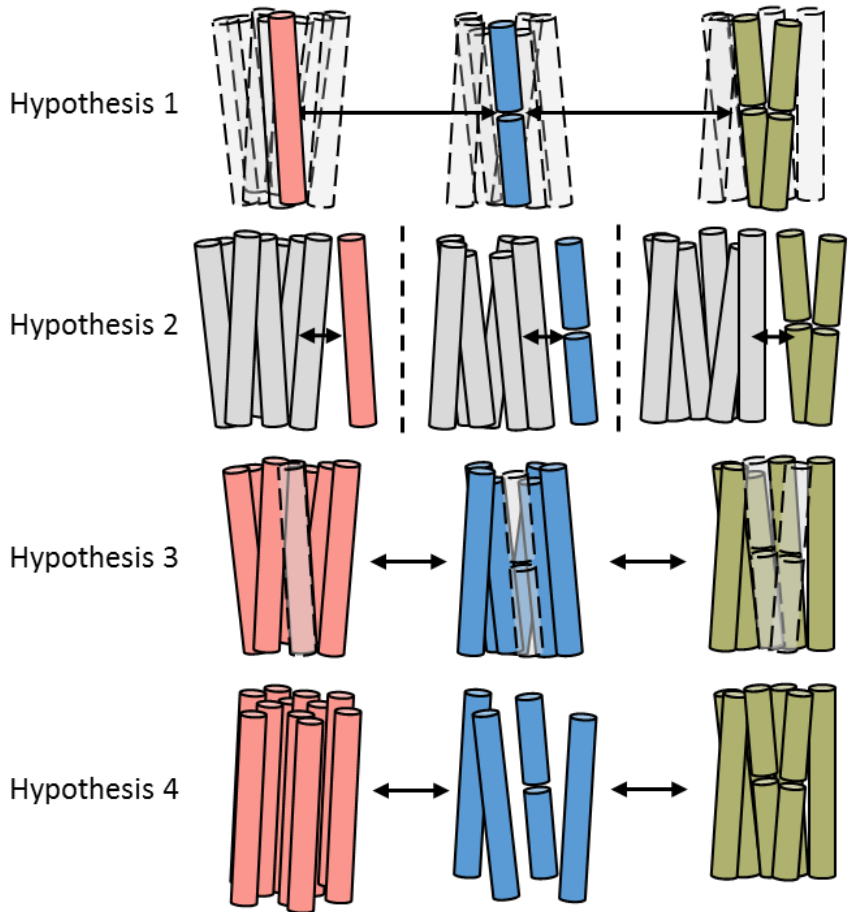

\section{$\int$}

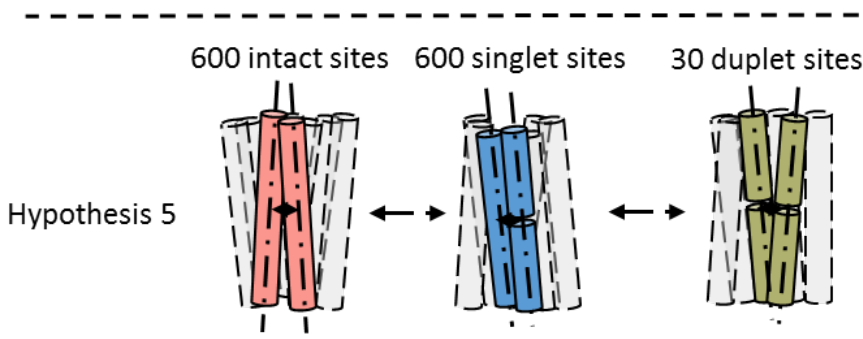

A nearest neighbouring distance analysis is performed, for which the central fibres are analysed together with their closest fibre

Fig. 6 The five comparisons are illustrated. Comparison 1 to 3 looks at the fibre orientation differences between the three classes of fibres, aiming at indications in the misorientation of the central broken fibres or the surrounding environments that can imply different morphologies for locally damaged sites. Comparison 4 accounts for the local fibre packing and misorientation scatter within sub-volumes. Lastly, comparison 5 investigates the nearest neighbouring distance between the same classes of fibres. 


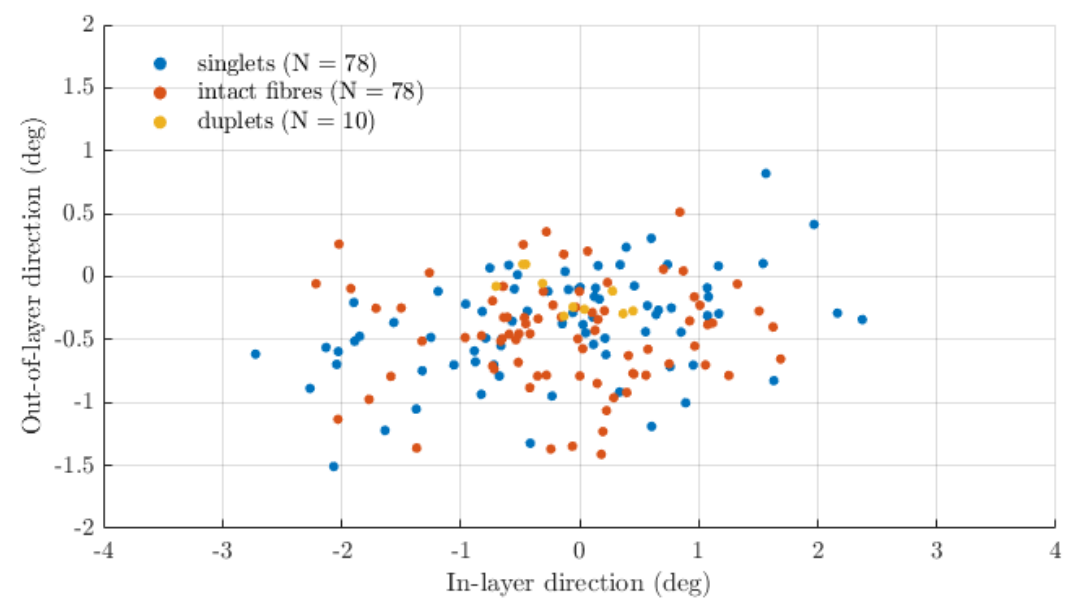

(a) Coupon 1

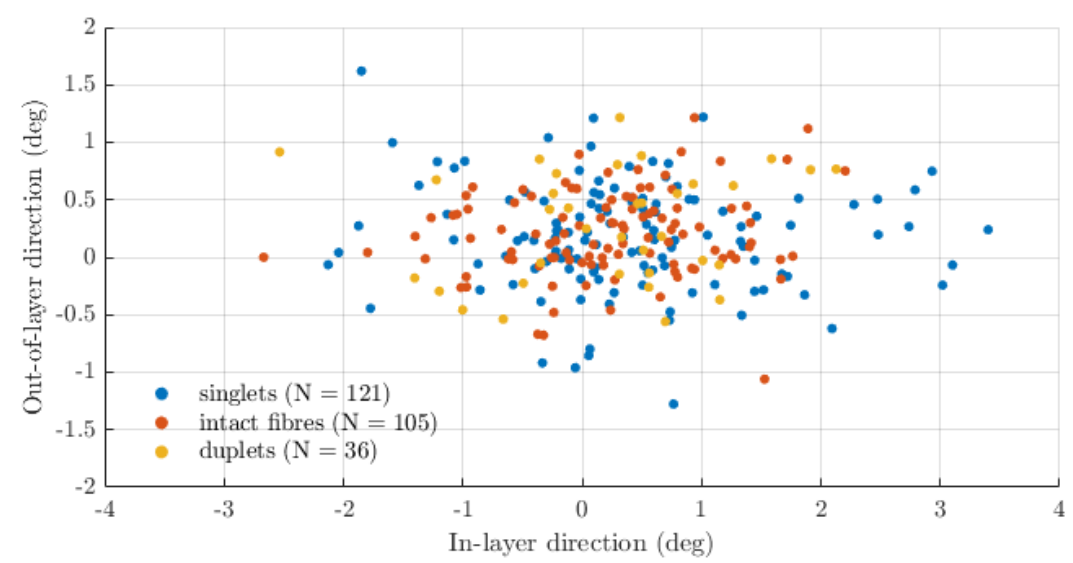

(b) Coupon 2

Fig. 7 Scatter plot of the fibre orientation of the individual fibres the sub-volumes at the intact, singlet and duplet sites.

Table 1. Hypothesis 1: the mean $(\mu)$ and standard deviation $(\sigma)$ of the intact and singlet fibres are shown for the in-layer direction. For both the assessed coupons, $\sigma$ of the singlet fibres is higher. In the two columns on the right, the Kolmogorov - Smirnov test results are indicated: the distributions of misorientations are drawn from a same population.

\begin{tabular}{cccccccc}
\hline \multirow{2}{*}{ Coupon } & \multicolumn{2}{c}{$\boldsymbol{\mu}$ misorientation (deg) } & \multicolumn{2}{c}{$\boldsymbol{\sigma}$ misorientation (-) } & \multirow{2}{*}{ K-S test } & p-value \\
\cline { 2 - 5 } & $\begin{array}{c}\text { Intact } \\
\text { fibres }\end{array}$ & $\begin{array}{c}\text { Singlet } \\
\text { fibres }\end{array}$ & $\begin{array}{c}\text { Intact } \\
\text { fibres }\end{array}$ & $\begin{array}{c}\text { Singlet } \\
\text { fibres }\end{array}$ & value & $(-)$ \\
\hline $\mathbf{1}$ & -0.13 & -0.16 & 0.90 & 1.11 & 0 & 0.52 \\
\hline $\mathbf{2}$ & 0.22 & 0.38 & 0.86 & 1.09 & 0 & 0.73 \\
\hline
\end{tabular}




\subsection{Hypothesis 1:}

The misorientation angles for the in-layer and out-of-layer directions are presented in Fig. 7 for broken and non-broken fibres. In the first instance, it may be seen that the spread of fibre misorientation is distinctly wider in the in-layer direction. The maximum range of in-layer orientations of the unbroken fibres (i.e. randomly selected microstructure) is $\sim 4-5^{\circ}$, while outof-layer range is around $2^{\circ}$, the corresponding standard deviations are $\sim 0.4^{\circ}$ and $\sim 1.1^{\circ}$. Whilst samples were cut from the same as-manufactured plate, and care was taken to cut and align samples prior to testing, there is a slight divergence of alignment between the two: Coupon 1 presents a slight out-of-layer tilt in the order of $0.5^{\circ}$, whilst Coupon 2 shows an in-layer tilt from the loading direction in the order of $0.2^{\circ}$.

While by looking at the mean value $(\mu)$ of the intact and singlet fibre misorientation distributions no difference is evinced, a consistent larger value is observed for the standard deviatipe 2 2 b distributions of data has indicated that these are drawn from the same population. K-S test results with associated $p$-values are reported in

Table 2, where 0 indicates the distributions are drawn from the same population and 1 that the populations are statistically distinct, with a threshold probability of 0.05 .

Table 2. Summary of the resampling analysis, carried out to check whether the duplet smaller sample size affected the comparison results. $K-S$ and $A-D$ tests are performed before implementing the resampling approach. Each of the 1000 samples randomly extracted from the singlet and intact sites, is $K-S$ and $A-D$ tested and the proportion of values below the $5 \%$ p-value threshold is indicated.

\begin{tabular}{ccccc}
\hline \multirow{2}{*}{ Test performed } & \multicolumn{2}{c}{ Coupon 1 } & \multicolumn{2}{c}{ Coupon 2 } \\
\cline { 2 - 5 } & $\begin{array}{c}\text { Duplet } \\
\text { intact }\end{array}$ & $\begin{array}{l}\text { Duplet } \\
\text { singlet }\end{array}$ & $\begin{array}{l}\text { Duplet } \\
\text { intact }\end{array}$ & $\begin{array}{c}\text { Duplet } \\
\text { singlet }\end{array}$ \\
\hline K-S before resampling & 0.59 & 0.31 & 0.57 & 0.66 \\
\hline A-D before resampling & 0.44 & 0.18 & 0.90 & 0.80 \\
\hline K-S p-value proportion $<\mathbf{0 . 0 5}$ & 0.00 & 0.02 & 0.00 & 0.00 \\
\hline A-D p-value proportion $<\mathbf{0 . 0 5}$ & 0.03 & 0.09 & 0.00 & 0.00 \\
\hline
\end{tabular}




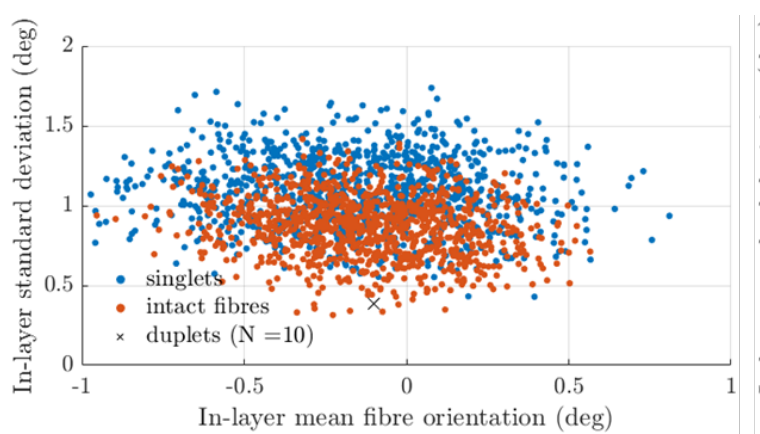

(a) Coupon 1

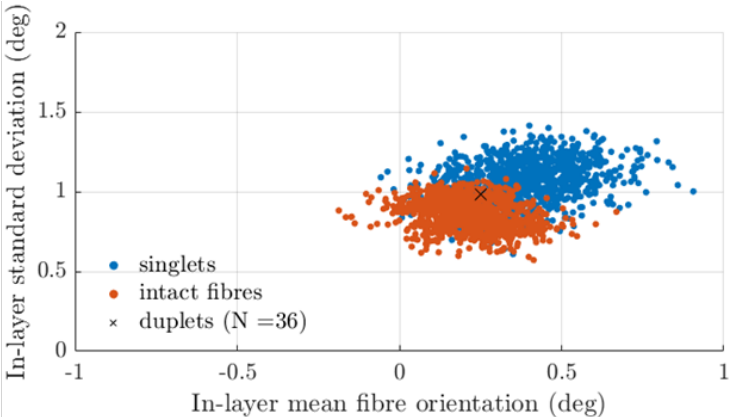

(b) Coupon 2

Fig. 8 Scatter plot of the 1000 resamples to the smallest sample dimension (duplet samples of respectively 10 and 36 measurements).

\subsubsection{Unbalanced sample size comparison statistical tests}

Given that the duplet fibres sample size is significantly smaller than the intact and singlet samples (across both coupons total duplets $N=46$, as opposed to $N \sim 200$ measured for intact fibres and singlets), sub-sampling of the singlet and intact fibre datasets to ensure an equal sample size in the K-S and A-D tests. Multiple random subsamples were considered, to account for variation in results between subsamples. Subsampling was used as statistical tests for unbalanced samples often result in bias. The same approach is implemented for all the comparisons presenting an unbalanced sample size.

The software environment for-statistical computing and graphics $R$ ([21]) is adopted to check whether the unbalanced sample sizes affect the comparison results. Both an Anderson Darling (A-D) and K-S tests are used, where A-D is more sensitive to the tails of the distributions than K$S$ [20]. The same statistical tests (K-S and A-D tests) are performed before and after carrying out the resampling to the smallest sample dimension, and results are compared. Resampling is performed by randomly extracting without replacement 1000 samples of data extracted from the intact and singlet sites of the dimension of the duplet sites, i.e. for coupon 1 and 2, 1000 samples made of respectively 10 and 36 values of singlet and intact fibre misorientation angles. Each sub-sample of data is compared to the sample of duplet fibre angles using the K-S and A-D tests. The proportion of p-values below 0.05 (indicating statistically significant difference between the distributions of the data) is reported in Table 2. For both coupons and comparisons (duplet to singlet fibres and duplet to intact fibres), the proportion below 0.05 is low enough to confirm that the smaller duplet sample size does not statistically affect the observed results: there is statistical evidence to suggest that the duplet fibre misorientation distribution is drawn from the same population of fibre misorientation as that of the singlet/intact fibres.

In Fig. 8, for coupon 1 and 2, the mean and standard deviation values for the extracted singlet and intact sub-samples and duplet samples is shown. As can be seen, the in-layer standard deviation is slightly higher for the singlet sites than for the intact sites.

\subsection{Hypothesis 2: broken fibres to their surrounding environment}

The fibre orientation of the singlet fibres and their surrounding environment is analysed. For coupon 1, 78 singlet fibre orientations are compared to the 2054 background surrounding fibres extracted from the same singlet sites (121 to 3400 for coupon 2), see Fig. 9. Given that the two samples of data are unbalanced, a resampling approach is adopted, as done for §4.1.1. The 
proportion of $p$-values of the 1000 extracted sub-samples is observed being about $1 \%$, providing no statistical evidence to suggest that the distributions of the fibre misorientations differ. The same comparison is performed for the duplet and the intact sites, showing no statistical evidence for a difference; and hence suggesting that the fibres broken in a duplet or singlet fashion do not have a different orientation if compared to their background environment.

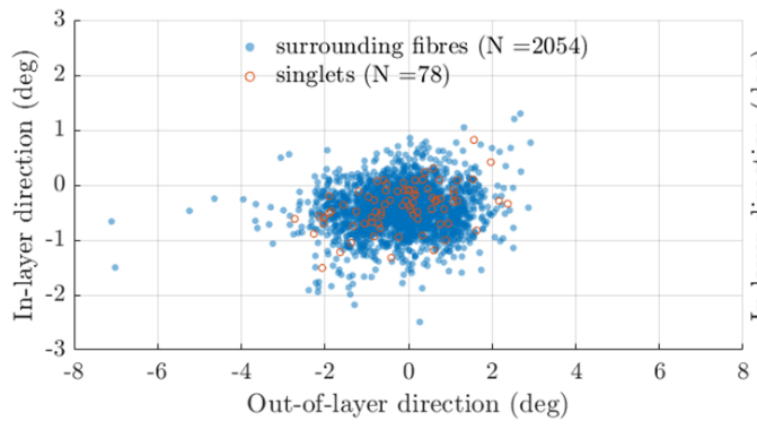

(a) Coupon 1

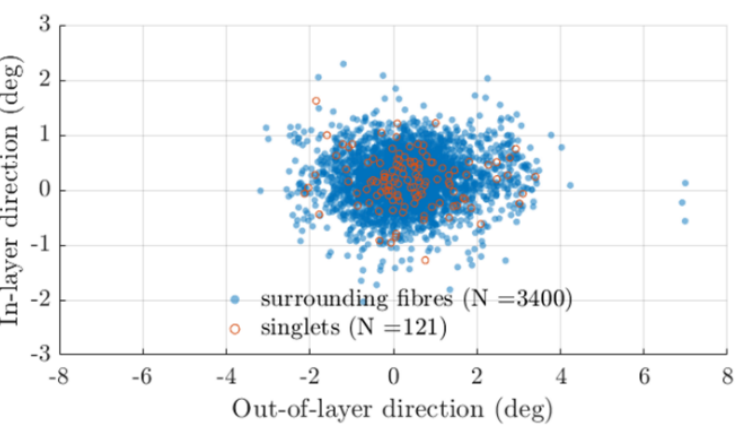

(b) Coupon 2

Fig. 9 For coupon 1 and 2, the misorientation angles in the in-layer and out-of-layer directions are plotted for the singlet fibres and the surrounding environment. The results of the $K$-S tests run for the distributions in the two separate directions are shown (zero meaning distributions are drawn from the same population).

\subsection{Hypothesis 3: the background environments}

The distributions of fibre orientations in the in-layer direction are compared for the singlet and intact background fibres (central fibres are excluded from the comparison). To better compare the misorientation values of the two coupons, the average fibre orientation (calculated considering all sites) of a single coupon is evaluated. This value is subtracted from each fibre misorientation value, providing the distributions are centred on the zero (representing the vertical loading direction). Coupon 2 appeared to be overall more misaligned in the in-layer direction and on the opposite direction of coupon 1 (by a fraction of degree). This could be introduced from the manufacturing of the coupon. For this reason, the signs of the values for coupon 2 are reversed in the histograms shown in Fig. 10. Having a lower number of measurements and to better visualise the different activities in the tails of the distributions, the duplet environment distributions are not included in the histogram plots. A K-S test carried out on the background fibre distributions of the singlet and intact sites provided statistical evidence to suggest that the distributions are drawn from different populations. Very similar results are obtained if comparing the duplet to the intact environment. The mean $\mu$ of the singlet and intact environments are different, as well as the standard deviation $\sigma$, which is fractionally higher in the singlet environment distribution (see Table 4 in §4.3.1.2).

\subsubsection{Analyses of the tails of the background environment distributions}

In the two following sections, statistical tools are implemented to better highlight dissimilarities between the distributions of data of the background environments. In §4.3.1.1, the outliers of the distributions are detected and removed to assess their influence on the 
statistical comparison. Following this, Kurtosis and Skewness analyses ([41]) are employed to further discern the distributions. These tests also allow a better definition of the length-scale over which patterns of fibre misorientation may or may not affect fibre break formation, as will be discussed in §4.3.1.2.

\subsubsection{Outliers detection and removal through the Pukelsheim three sigma rule}

An outlier detection technique is adopted to better understand if the differences observed for $\mu$ and $\sigma$ in $\S 4.3$ are related to the different tails of the distributions. The aim is to assess the impact of outliers on the results of the K-S test and A-D test and the standard deviations of the samples of data. Outliers can be detected in a number of different ways. In this study, a Pukelsheim three sigma rule is used to detect the outliers [22]. This rule states that $95 \%$ of the density of unimodal distributions lies within three standard deviations of the mean, hence the outliers are treated as those which fall outside this range. Initial analysis of this dataset suggests that the distribution of misorientation data is unimodal. If removing these values makes a difference to the analysis, then the outliers should be checked to identify if they are errors or points of interest.

For the in-layer directions, two comparisons are made: duplet to intact and singlet to intact environments.

Table 3 shows the results for the first three width steps to show how values change when moving from width 2 to thicker or thinner cross-sections, i.e. including more or less fibres surrounding a broken one.

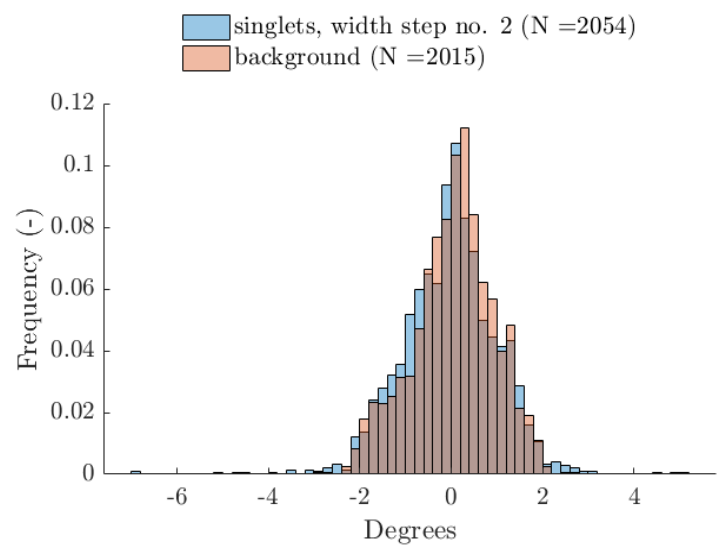

(a) Coupon 1

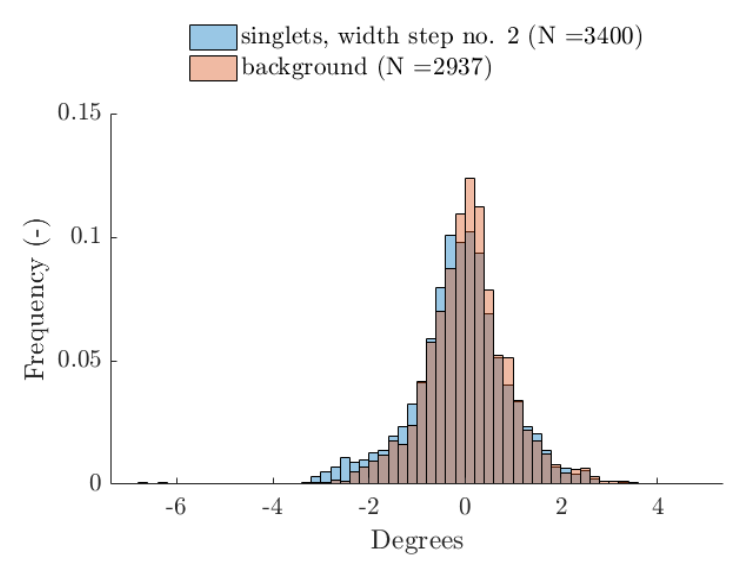

(a) Coupon 2

Fig. 10 The distributions of the misorientation angles on the in-layer direction are shown.

To better compare the misorientation values of the two coupons, the distributions are shifted of the average coupon fibre orientation. For coupon 2, the sign of the orientation values is 
inverted to better compare the two distributions. Statistical assessments are made using the original distributions of data.

Table $3 \mathrm{~K}-\mathrm{S}$ and $\mathrm{A}-\mathrm{D}$ tests to estimate dissimilarities between the distributions of fibre misorientation before and after removal of outliers using the Pukelsheim three sigma rule. A pvalue below 0.05 represents a statistically significant difference between the compared distributions.

\begin{tabular}{ccccccc}
\hline $\begin{array}{c}\text { Singlet to intact } \\
\text { Duplet to intact }\end{array}$ & \multicolumn{3}{c}{ Coupon 1 } & & & Coupon 2 \\
\hline Width & 1 & 2 & 3 & 1 & 2 & 3 \\
\hline K-S p-value before & 0.01 & 0.00 & 0.00 & 0.00 & 0.00 & 0.00 \\
\cline { 2 - 7 } outliers removal & 0.20 & 0.10 & 0.02 & 0.12 & 0.03 & 0.02 \\
\hline A-D p-value before & 0.01 & 0.00 & 0.00 & 0.00 & 0.00 & 0.00 \\
\cline { 2 - 7 } outliers removal & 0.08 & 0.02 & 0.00 & 0.09 & 0.03 & 0.04 \\
\hline Number of outliers & 4,1 & 13,7 & 17,24 & 8,14 & 16,27 & 27,36 \\
\cline { 2 - 7 }$\quad$ detected & 1,1 & 2,7 & 2,24 & 0,14 & 4,27 & 4,36 \\
\hline K-S p-value after & 0.01 & 0.00 & 0.00 & 0.00 & 0.00 & 0.00 \\
\hline outliers removal & 0.22 & 0.05 & 0.01 & 0.13 & 0.04 & 0.02 \\
\hline A-D p-value after & 0.01 & 0.00 & 0.00 & 0.00 & 0.00 & 0.00 \\
\cline { 2 - 7 } outliers removal & 0.06 & 0.01 & 0.00 & 0.08 & 0.03 & 0.03 \\
\hline
\end{tabular}

In 
Table 3, the number of outliers removed from the left and the right side of the distributions are indicated. The K-S and A-D tests are used to compare the distributions before and after the outlier removal. For all the analysed cases, the $\mathrm{p}$-values of the K-S and A-D tests before and after outlier removal reveal no evidence of outliers effects on the results of the statistical tests. No firm conclusions can be drawn from the comparison between duplet and intact sites, with width 3 being the only step at which statistical differences between the distributions are observed for both coupons. This can be related to the particularly low number of duplet sites available. As to the singlet to intact environment comparison, for both coupons it is there is statistical evidence to suggest that the two distributions are drawn from different populations, according to both K-S and A-D tests, before and after outlier removal, meaning the difference between the background environments of singlet and intact sites cannot be attributed to the extreme values of the distributions but there is evidence to suggest it is related to an intrinsic dissimilarity.

\subsubsection{Kurtosis and Skewness analyses and justification of the length-scale choice}

Kurtosis is a measure of how outlier-prone a distribution is. Where the kurtosis of the normal distribution is 3 , distributions that are more outlier-prone than the normal distribution have kurtosis greater than 3 and distributions that are less outlier-prone have kurtosis less than 3 . As shown in Fig. 11, with the exception of width 1, all the analysed widths provide distributions of fibre misorientation that are more outlier-prone than a normal distribution.

Skewness is a measure of the symmetry of a distribution. The normal distribution is a symmetric distribution, and its skewness value is zero. A negative skewness value means the distribution is asymmetrical and its values are skewed to values below the mean. The opposite is valid for positive skewness values [41,42]. Of all the analysed width steps, width 2 shows the greatest difference between the kurtosis values. This lead us to give more interest to width 2, being the area around the central fibres at which the difference between damaged and intact sites was the greatest. Results are shown for coupon 1 which of the two coupons, is the most aligned to the loading direction. The results of the Skewness analysis indicate how the singlet background fibre distribution is slightly more skewed left on widths 2, 3 and 4. In Table 4, the results of the $\mathrm{K}$-S tests for both coupons, surrounding environment fibres distribution in the in-layer direction are shown. With a $p$-value equal to 0 , there is strong statistical evidence to suggest that the two distributions representing the environments in the singlet and intact sites are different. 


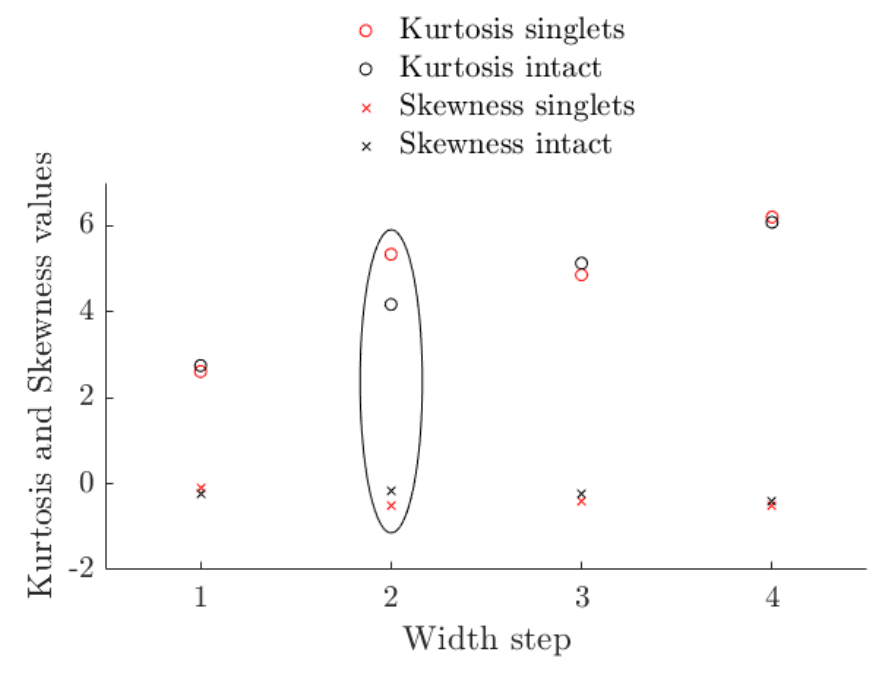

Fig. 11 The results of the Kurtosis and Skewness analyses are shown for coupon 1. Of all the analysed width steps, width 2 shows the greatest difference between results.

Table 4 Hypothesis 3: $\mu$ and $\sigma$ of the intact and singlet environment fibres are shown for the in-layer direction. For both the assessed coupons, $\sigma$ of the singlet fibres is higher. In the two columns on the right, the Kolmogorov - Smirnov test results are indicated: the distributions of misorientations are proven to be statistically different with a p-value equal to zero.

\begin{tabular}{ccccccc}
\hline \multirow{2}{*}{ Coupon } & \multicolumn{2}{c}{$\boldsymbol{\mu}$ misorientation (deg) } & \multicolumn{2}{c}{$\boldsymbol{\sigma}$ misorientation $(-)$} & \multirow{2}{*}{ K-S test } & p-value \\
\cline { 2 - 5 } & Intact fibres & $\begin{array}{c}\text { Singlet } \\
\text { fibres }\end{array}$ & $\begin{array}{l}\text { Intact } \\
\text { fibres }\end{array}$ & $\begin{array}{c}\text { Singlet } \\
\text { fibres }\end{array}$ & value & $(-)$ \\
\hline $\mathbf{1}$ & -0.09 & -0.18 & 0.92 & 1.01 & 1 & 0 \\
\hline $\mathbf{2}$ & 0.18 & 0.33 & 0.92 & 1.01 & 1 & 0 \\
\hline
\end{tabular}

\subsection{Hypothesis 4: Local fibre packing and misorientation scatter within sub-volumes}

When a fibre breaks, the surrounding fibres experience a stress increase. This is quantified using the stress concentration factor (SCF), which is the relative increase in stress these fibres will perceive [6]. Fibre packing affects the SCF and starting from the 1960's modellers have considered different fibre arrangements moving from a regular 1D to a random 2D configuration and quantified the effect of these packing arrangements on the SCF values [43-45]. Using a finite element approach, Swolfs et al. showed random packing leads to higher SCF values if compared to regular arrangements [46]. This provides further significance to the examination of the local fibre packing in the analysed coupons to understand if fibre failure is affected by local volumetric fibre distribution.

Only fibres fully contained within the analysed volume (of width 2 cross-section described in §3.1.2) were tracked in this study. Hence, the parameter presented below will be identified as a fibre volume fraction 'factor', obtained as a classic fibre volume fraction value but not accounting for portions of fibres falling within the edges of volume, i.e. this factor is equal to or lower than 
the fibre volume fraction. In Fig. 12, for coupon 2 (presenting the highest number of sites) the factor is plotted against the standard error of the misorientation of the fibres comprised in a subvolume. Each point is indicative of a full sub-volume condition. A sub-volume with a higher fraction factor is expected to have a lower misorientation standard error due to the fibres being closely packed together, where the opposite is likely to happen for a sub-volume with a low fraction factor.

The mean and the standard deviation of the values for the three sub-volume categories are reported in

Table 5, highlighting that no major differences are observed. K-S tests run separately on the two parameters comparing the distributions singlet-intact, singlet-duplet and intact-duplet suggest no statistical differences can be evinced ( $p$-values in the range of $0.5-0.7)$. Similar results are obtained for coupon 1.

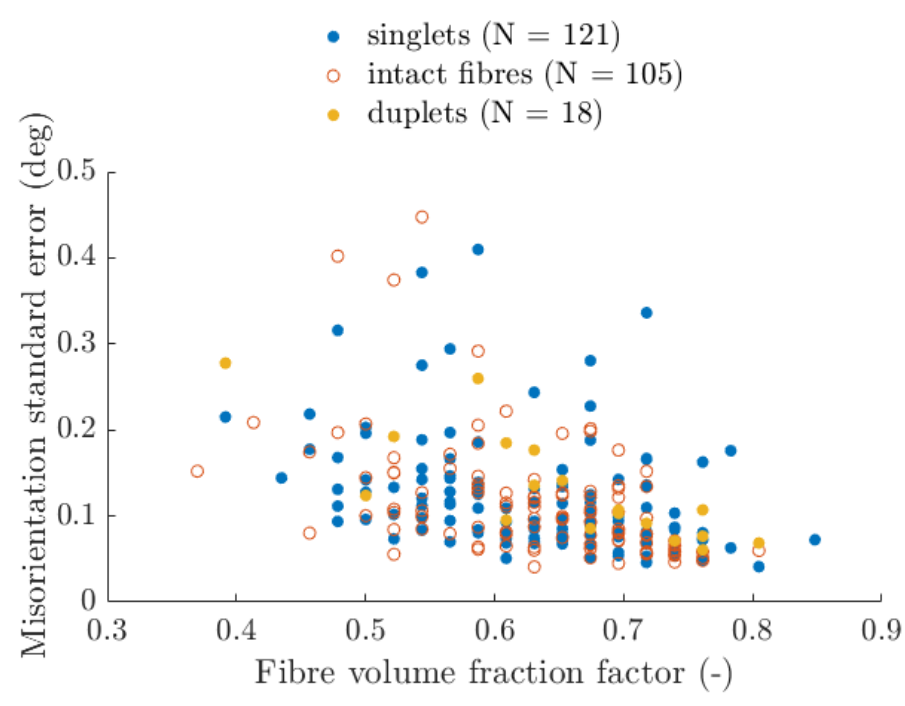

Fig. 12 The sub-volumes fibre volume fraction factors (number of entire fibres per sub-volume cross-sectional area times the average nominal fibre cross-section) against the standard error of the misorientation of the fibres within the sub-volumes of coupon 2 (showing the highest number of sub-volumes), width 2. $\mathrm{K}$-S tests show no statistical differences between the three categories. Similar results are obtained for coupon 1.

Table $5 \mu$ and $\sigma$ of the fibre fraction factor and misorientation standard error shown in Fig. 12. No statistical differences are evinced among the three site categories.

\begin{tabular}{ccccccc}
\hline \multicolumn{4}{c}{$\boldsymbol{\mu}$} & \multicolumn{3}{c}{$\boldsymbol{\sigma}$} \\
\cline { 2 - 6 } & Intact & Singlet & Duplet & Intact & Singlet & Duplet \\
fibres & fibres & fibres & fibres & fibres & fibres \\
\hline
\end{tabular}




\begin{tabular}{ccccccc}
\hline Fibre fraction factor (-) & 0.63 & 0.63 & 0.65 & 0.08 & 0.09 & 0.11 \\
\hline Misorientation st. error (-) & 0.12 & 0.12 & 0.13 & 0.07 & 0.07 & 0.06 \\
\hline
\end{tabular}

\subsection{Hypothesis 5: Nearest neighbour distance analysis}

A statistical assessment of the nearest-neighbour distances is carried out. Two different break clusters patterns are observed. In Fig. $13(a, b)$ an example of planar clusters and diffuse clusters is shown. Planar (or adjacent) clusters (focus of the study) are considered as groups of fibre breaks with a distance less than a fibre diameter. Fibre breaks with a distance less than the ineffective length are catalogued as diffuse clusters [6,28]. In Fig. 13 (c-f), examples of planar clusters are presented. As shown, clusters arise both in locally high volume fraction regions, and mixed regions, where a linear pattern is commonly observed. Except for two cases, fibre break clusters have not been observed to grow under increased loading, as illustrated in $[11,28,36]$. With a distance of 1.65 fibre diameter, the only case of a large nearest-neighbouring distance between two planar broken fibres is shown in Fig. 14.

This experimental finding confirms what would be intuitively indicated by a load shedding behaviour: clustering should happen mostly between close neighbours (higher load is seen by an intact fibre that is closer to a break). In Fig. 15, the nearest neighbouring fibre distance values collected for the two coupons is shown. For the singlet and intact locations, about 600 values have been collected, against 25 values for the duplet sites (related to the highest number of clusters observed in the prior-to-failure scans). With a few cases of fibres having the nearest neighbour at a distance greater than 1.5 fibre diameter, the mean nearest neighbouring distance observed value is for all the three cases of $\sim 1.1$ fibre diameter, meaning that even in low packed regions, the great majority of fibres tend to have at least one very close nearest neighbour. This is in contrast to 1.35 fibre diameter, the nearest neighbouring distance that is calculated assuming a $2 \mathrm{D}$ hexagonal packing with same fibre volume fraction (of $50 \%$ in this study) and according to that indicated by Swolfs et al. [6]: having random packings much closer fibres to the broken ones, these see an increase in the stress concentration factors when compared to regular packings. A K-S test is performed to compare the three groups of data, and it suggests that there are no statistical differences between the distributions shown in Fig. 15. 

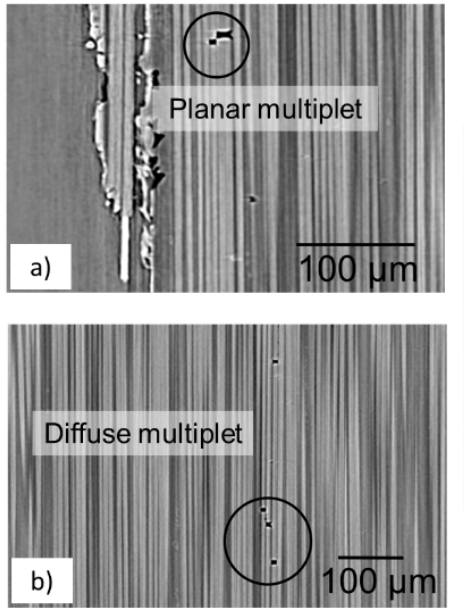
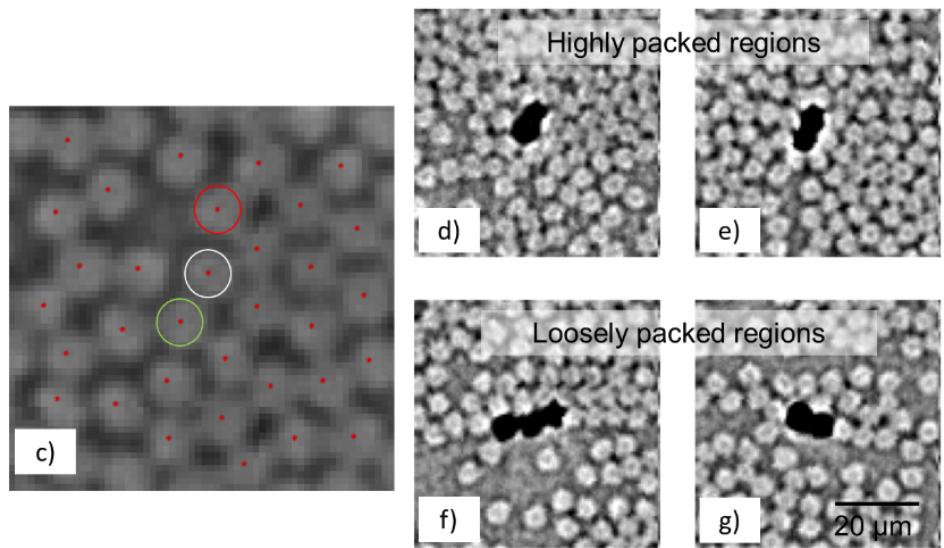

Fig. 13 (a) Example of a planar multiplet, (b) diffuse multiplet. In (c): in the white circle the fibre breaking in a duplet fashion. Circled in green its closest fibre, preferred for duplet formation; among all the neighbours, in red the furthest (typically not involved in multiplet formation) is highlighted. In $(d, e)$, planar duplets in a high fibre volume fraction region whereas in $(f, g)$ triplet and duplet are shown in a 'mixed' volume fraction region.
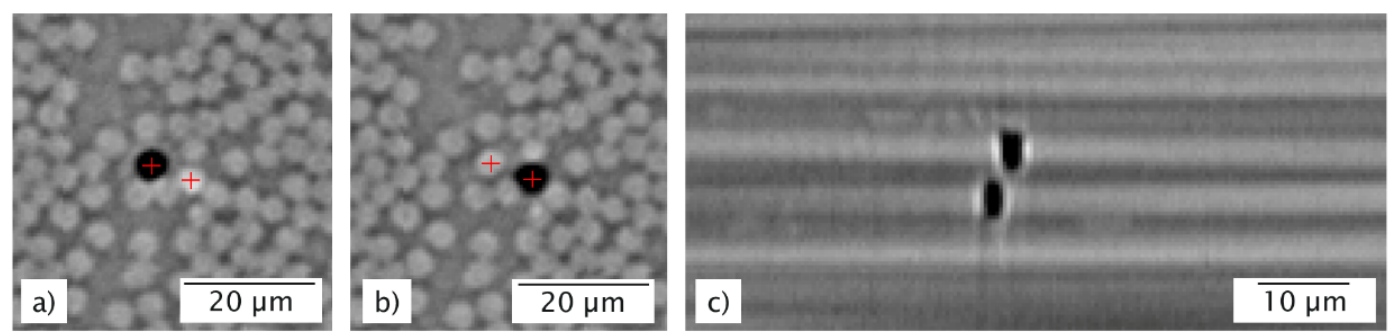

Fig. $14(a, b)$ Top view and (c) lateral view of the duplet with the largest detected distance observed in the two analysed coupons. Separation distance between the centres of the break was of $\sim 3 \mu \mathrm{m}$. 


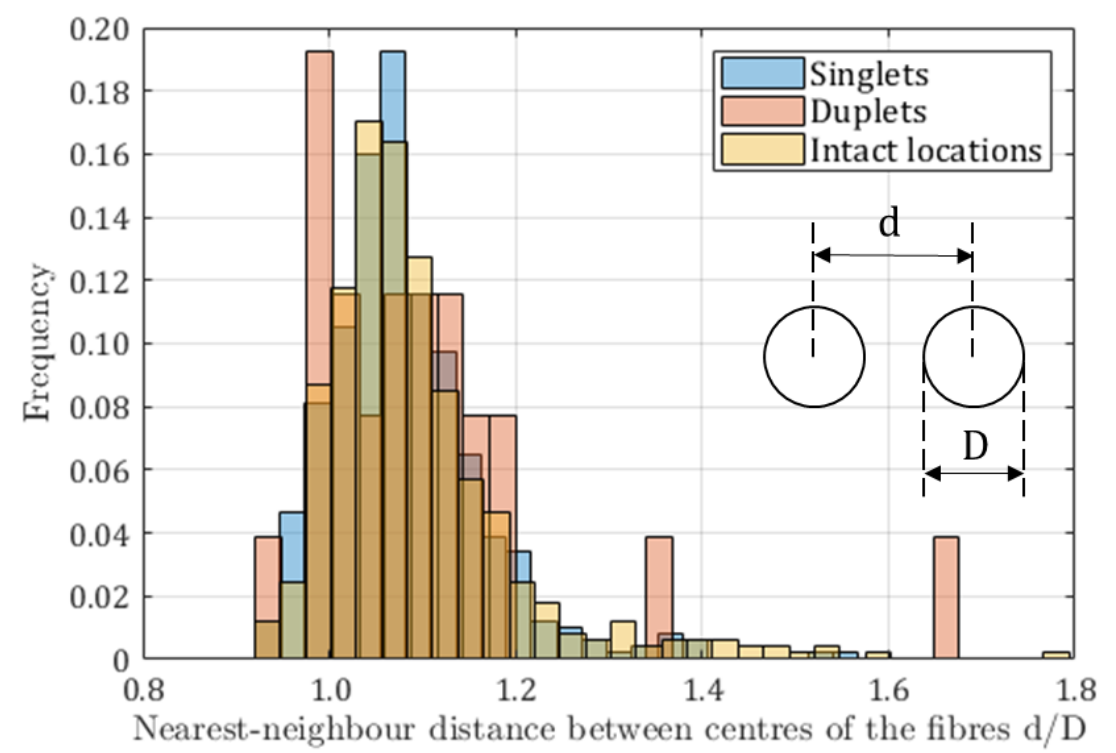

Fig. 15 Histogram of the relative distances between two broken fibres recorded a fibre diameter below the failure plane (duplets); relative distances between a broken fibre to its nearest neighbouring intact fibre (singlets); closest distance between random intact fibres (intact locations). Values are obtained combining the data of the two analysed coupons, cut from the same CFRP panel.

\section{Conclusions}

In situ synchrotron X-ray computed tomography was performed to investigate the morphology of local damaged sites in untoughened aerospace grade carbon-epoxy coupons under quasi-static tensile loading. It is well known that carbon fibre tensile strength distributions follow a Weibull probabilistic model. However, local fibre misorientation and packing needs to be assessed to clarify their effects on the failure processes.

To the best of the authors knowledge, this is the first time an automated approach was developed that uses image analysis and statistical tools to investigate at a micro-scale level single and adjacently coupled fibre break locations, assessing differences in the misorientation of the fibres and in the nearest neighbouring distances between fibres, both in damaged and nondamaged local sites. Great attention is given to the quality of the fibres segmented from low contrast images with high fibre volume fraction, with a detailed error trapping approach.

Statistically assessed experimental observations of the fibre misorientation angle distributions provide subtle indications on the existence of different morphologies among these sites. For the carbon fibre pre-preg composite coupons object of this study, there is statistical evidence to suggest that the local background environment of singlet sites and intact sites differ, providing input for further investigations. The single broken fibre orientation distribution shows a consistent higher standard deviation value, when compared to the intact fibre orientation distribution. In contrast, no statistically significant differences are observed regarding the fibre packing in locally damaged sites. The nearest neighbouring distances are evaluated for the cases of two intact fibres, one broken and one intact fibre and two adjacent broken fibres, showing that two fibres prefer to break in a nearest neighbouring fashion. These observations suggest that the morphology of fibre break sites appear as complex. By a simple visual assessment of a few sites, contrasting and incorrect conclusions could be reached, e.g. a fibre is more likely to 
fail if surrounded by misoriented, hence less loaded fibres or fibres break in a duplet fashion when close to each other and near a resin rich region, experiencing a higher stress than expected.

It is suggested in the literature that the singlet density is not likely to be the cause of longitudinal final failure of composites $[6,11,28]$, while the large multiplets might have a stronger role instead, suggesting a failure criterion driven by the Fracture Mechanics could be preferred over the Strength of Materials [2]. However, reliable models predicting both the damage development and the final failure of unidirectional composites should include and correctly describe the mechanisms leading to the accumulation of these features, that strongly escalates prior to final failure. The fibre strength is typically described using the Weibull strength distribution. However, measuring the Weibull distributions remains challenging and studies have shown that better distributions, more challenging to implement in models but more suitable to describe the strength variability could be adopted $([47,48])$. Hence, model overestimations in the singlet prediction are often attributed to the distribution errors, as done in [28]. However, by combining:

- in situ SRCT, allowing the damage to be fully captured in 3D in a prior-to-failure condition;

- automated image processing techniques, that enable the comprehensive morphology of damaged and non-damaged local regions to be extracted in low computational times from CT scans, with an error trapping approach that reduces the fibre centre detection error to a negligible level;

- reliable statistical tools, such as Kolmogorov-Smirnov tests, Anderson-Darling tests, Kurtosis and Skewness tests as well as a Pukelsheim three sigma rule, used to capture subtle differences in the distributions of the data extracted from self-similar microstructures difficult to discern by eye, recognise the variability in the sizes of the distributions of data and investigate the influence of the tails of the distributions, typically indication of errors or peculiar cases of interest;

it can now be suggested that the local morphology might have a role in the formation of fibre breaks as it is shown that statistically the background environment of singlet sites has some peculiarities that should be further explored by including the real fibre geometry as extracted from the analysed sites into finite element (FE) predictive models.

This paper represents a first step towards the final aim of the study, which consists of providing a full characterisation of the large multiplet local morphology that can provide the modelling community more information on the influence that these features have on the final failure events. However, larger experimental campaigns are needed that test a greater amount of coupons of the same material, as in this study a total of four 4-plets have been captured in the prior-to-failure scans from the two analysed coupons, i.e. tens of coupons should be tested to obtain statistically approachable data.

The tools are now developed, with a high level of confidence in the basic measurements and other application areas can be envisaged where micro-structural influence may be important: e.g. compression/microbuckling, and materials with non-ideal unidirectional alignment. In addition to the applications on particular materials, the work presented in this paper illustrates an approach, consistent with the philosophy of "data rich mechanics" whereby very high levels of utilisation of experimental studies can be used to extract data by which to inform model development and validation. 


\section{Acknowledgements}

The authors would like to acknowledge the European Synchrotron Radiation Facility for provision of synchrotron radiation facilities and would like to thank Dr Lukas Helfen and Ms Elodie Boller for assistance in using beamline ID19. The authors also gratefully acknowledge Mitsubishi Chemical Co. for materials supply and the $\mu$-VIS X-Ray Imaging Centre at the University of Southampton for provision of tomographic imaging facilities, supported by EPSRC grant EP-H01506X. 
[1] Y. Swolfs, I. Verpoest, L. Gorbatikh, Issues in strength models for unidirectional fibrereinforced composites related to Weibull distributions, fibre packings and boundary effects, Compos. Sci. Technol. 114 (2015) 42-49. doi:10.1016/j.compscitech.2015.04.002.

[2] A. Bunsell, L. Gorbatikh, H. Morton, S. Pimenta, I. Sinclair, M. Spearing, Y. Swolfs, A. Thionnet, Benchmarking of strength models for unidirectional composites under longitudinal tension, Compos. Part A Appl. Sci. Manuf. 111 (2018) 138-150. doi:10.1016/j.compositesa.2018.03.016.

[3] P.D. Soden, A.S. Kaddour, M.J. Hinton, Recommendations for designers and researchers resulting from the world-wide failure exercise, Fail. Criteria Fibre-Reinforced-Polymer Compos. 64 (2004) 1223-1251. doi:10.1016/B978-008044475-8/50039-1.

[4] A.S. Kaddour, M.J. Hinton, P.A. Smith, S. Li, The background to the third world-wide failure exercise, J. Compos. Mater. 47 (2013) 2417-2426. doi:10.1177/0021998313499475.

[5] A.S. Kaddour, M.J. Hinton, P.A. Smith, S. Li, The background to the third world-wide failure exercise, J. Compos. Mater. 47 (2013) 2417-2426. doi:10.1177/0021998313499475.

[6] Y. Swolfs, I. Verpoest, L. Gorbatikh, A review of input data and modelling assumptions in longitudinal strength models for unidirectional fibre-reinforced composites, Compos. Struct. 150 (2016) 153-172. doi:10.1016/j.compstruct.2016.05.002.

[7] J.M. Hedgepeth, Stress Concentrations In Filamentary Structures, NASA Tech. Note D882. (1961) 36.

[8] C. Zweben, B.W. Rosen, A statistical theory of material strength with application to composite materials, J. Mech. Phys. Solids. 18 (1970) 189-206. doi:10.1016/00225096(70)90023-2.

[9] I.J. Beyerlein, S.L. Phoenix, Statistics for the strength and size effects of microcomposites with four carbon fibers in epoxy resin, Compos. Sci. Technol. 56 (1996) 75-92. doi:10.1016/0266-3538(95)00131-X.

[10] B. Rosen, N. Dow, Z. Hashin, Mechanical Properties of Fibrous Composites., (1964). http://oai.dtic.mil/oai/oai?verb=getRecord\&metadataPrefix=html\&identifier=ADA3082 19.

[11] A.E. Scott, M. Mavrogordato, P. Wright, I. Sinclair, S.M. Spearing, In situ fibre fracture measurement in carbon-epoxy laminates using high resolution computed tomography, Compos. Sci. Technol. 71 (2011) 1471-1477. doi:10.1016/j.compscitech.2011.06.004.

[12] S. Behzadi, P.T. Curtis, F.R. Jones, Improving the prediction of tensile failure in unidirectional fibre composites by introducing matrix shear yielding, Compos. Sci. Technol. 69 (2009) 2421-2427. doi:10.1016/j.compscitech.2009.06.010.

[13] S. Pimenta, A computationally-efficient hierarchical scaling law to predict damage accumulation in composite fibre-bundles, Compos. Sci. Technol. 146 (2017) 210-225. doi:10.1016/j.compscitech.2017.04.018.

[14] T. Okabe, H. Sekine, K. Ishii, M. Nishikawa, N. Takeda, Numerical method for failure simulation of unidirectional fiber-reinforced composites with spring element model, Compos. Sci. Technol. 65 (2005) 921-933. doi:10.1016/j.compscitech.2004.10.030.

[15] T. Okabe, M. Nishikawa, N. Takeda, H. Sekine, Effect of matrix hardening on the tensile strength of alumina fiber-reinforced aluminum matrix composites, Acta Mater. 54 (2006) 2557-2566. doi:10.1016/j.actamat.2006.01.044.

[16] G. Yamamoto, M. Onodera, K. Koizumi, J. Watanabe, H. Okuda, F. Tanaka, T. Okabe, Considering the stress concentration of fiber surfaces in the prediction of the tensile strength of unidirectional carbon fiber-reinforced plastic composites, Compos. Part A Appl. Sci. Manuf. 121 (2019) 499-509. doi:10.1016/j.compositesa.2019.04.011.

[17] S.J. Zhou, W.A. Curtin, Failure of fiber composites: A lattice green function model, Acta Metall. Mater. (1995). doi:10.1016/0956-7151(95)00003-E. 
[18] R.P. Tavares, F. Otero, A. Turon, P.P. Camanho, Effective simulation of the mechanics of longitudinal tensile failure of unidirectional polymer composites, Int. J. Fract. 208 (2017) 269-285. doi:10.1007/s10704-017-0252-9.

[19] R.P. Tavares, F. Otero, J. Baiges, A. Turon, P.P. Camanho, A dynamic spring element model for the prediction of longitudinal failure of polymer composites, Comput. Mater. Sci. 160 (2019) 42-52. doi:10.1016/j.commatsci.2018.12.048.

[20] Y. Swolfs, R.M. McMeeking, I. Verpoest, L. Gorbatikh, Matrix cracks around fibre breaks and their effect on stress redistribution and failure development in unidirectional composites, Compos. Sci. Technol. 108 (2015) 16-22. doi:10.1016/j.compscitech.2015.01.002.

[21] Y. Swolfs, I. Verpoest, L. Gorbatikh, Maximising the hybrid effect in unidirectional hybrid composites, Mater. Des. 93 (2016) 39-45. doi:10.1016/j.matdes.2015.12.137.

[22] H.Y. Chou, A.R. Bunsell, G. Mair, A. Thionnet, Effect of the loading rate on ultimate strength of composites. Application: Pressure vessel slow burst test, Compos. Struct. 104 (2013) 144-153. doi:10.1016/j.compstruct.2013.04.003.

[23] H.Y. Chou, A. Thionnet, A. Mouritz, A.R. Bunsell, Stochastic factors controlling the failure of carbon/epoxy composites, J. Mater. Sci. 51 (2016) 311-333. doi:10.1007/s10853-0159390-5.

[24] a. Thionnet, H.Y. Chou, a. Bunsell, Fibre Break Failure Processes in Unidirectional Composites. Part 3: Unidirectional Plies Included in Laminates, Appl. Compos. Mater. 22 (2014) 157-169. doi:10.1007/s10443-014-9399-y.

[25] a. Thionnet, H.Y. Chou, A. Bunsell, Fibre Break Failure Processes in Unidirectional Composites. Part 1: Failure and Critical Damage State Induced by Increasing Tensile Loading, Appl. Compos. Mater. 22 (2014) 141-155. doi:10.1007/s10443-014-9398-z.

[26] a. Thionnet, H.Y. Chou, a. Bunsell, Fibre Break Failure Processes in Unidirectional Composites. Part 2: Failure and Critical Damage State Induced by Sustained Tensile Loading, Appl. Compos. Mater. 22 (2014) 141-155. doi:10.1007/s10443-014-9398-z.

[27] L. St-Pierre, N.J. Martorell, S.T. Pinho, Stress redistribution around clusters of broken fibres in a composite, Compos. Struct. 168 (2017). doi:10.1016/j.compstruct.2017.01.084.

[28] Y. Swolfs, H. Morton, A.E. Scott, L. Gorbatikh, P.A.S. Reed, I. Sinclair, S.M. Spearing, I. Verpoest, Synchrotron radiation computed tomography for experimental validation of a tensile strength model for unidirectional fibre-reinforced composites, Compos. Part A Appl. Sci. Manuf. 77 (2015) 106-113. doi:10.1016/j.compositesa.2015.06.018.

[29] T.W.C. C.J. Creighton, M.P.F. Sutcliffe, A multiple field image analysis procedure for characterisation of fibre alignment in composites, Selenium Its Mol. Biol. Role Hum. Heal. 9781461410 (2012) 335-344. doi:10.1007/978-1-4614-1025-6_26.

[30] M.W. Czabaj, M.L. Riccio, W.W. Whitacre, Numerical reconstruction of graphite/epoxy composite microstructure based on sub-micron resolution X-ray computed tomography, Compos. Sci. Technol. (2014). doi:10.1016/j.compscitech.2014.10.017.

[31] M.J. Emerson, K.M. Jespersen, A.B. Dahl, K. Conradsen, L.P. Mikkelsen, Individual fibre segmentation from 3D X-ray computed tomography to study the misalignment in unidirectional composite materials, Compos. Part A Appl. Sci. Manuf. 97 (2016) 83-92. doi:10.1016/j.compositesa.2016.12.028.

[32] M.J. Emerson, Y. Wang, P.J. Withers, K. Conradsen, A.B. Dahl, V.A. Dahl, Quantifying fibre reorientation during axial compression of a composite through time-lapse $\mathrm{X}$-ray imaging and individual fibre tracking, Compos. Sci. Technol. 168 (2018) 47-54. doi:10.1016/j.compscitech.2018.08.028.

[33] N. Otsu, A threshold selection method from gray-level histograms, IEEE Trans. Syst. Man. Cybern. C (1979) 62-66. doi:10.1109/TSMC.1979.4310076.

[34] S.C. Garcea, Y. Wang, P.J. Withers, X-ray computed tomography of polymer composites, Compos. Sci. Technol. 156 (2018) 305-319. doi:10.1016/j.compscitech.2017.10.023. 
[35] P. Wright, X. Fu, I. Sinclair, S.M. Spearing, Ultra High Resolution Computed Tomography of Damage in Notched Carbon Fiber--Epoxy Composites, J. Compos. Mater. 42 (2008) 1993-2002. doi:10.1177/0021998308092211.

[36] S.C. Garcea, I. Sinclair, S.M. Spearing, P.J. Withers, Mapping fi bre failure in situ in carbon fi bre reinforced polymers by fast synchrotron X-ray computed tomography, Compos. Sci. Technol. 149 (2017) 81-89. doi:10.1016/j.compscitech.2017.06.006.

[37] S.C. Garcea, M.N. Mavrogordato, A.E. Scott, I. Sinclair, S.M. Spearing, Fatigue micromechanism characterisation in carbon fibre reinforced polymers using synchrotron radiation computed tomography, Compos. Sci. Technol. 99 (2014) 23-30. doi:10.1016/j.compscitech.2014.05.006.

[38] W.S. Rasband, ImageJ, U. S. National Institutes of Health, Bethesda, Maryland, USA, 1997. https://imagej.nih.gov/ij/.

[39] C.M. Thompson, L. Shure, Image Processing Toolbox: For Use with MATLAB; [user's Guide], MathWorks, 1995.

[40] T.B. Arnold, J.W. Emerson, Nonparametric Goodness-of-Fit Tests for Discrete Null Distributions, (n.d.) 34-39.

[41] H.-Y. Kim, Statistical notes for clinical researchers: assessing normal distribution (2) using skewness and kurtosis, Restor. Dent. Endod. 38 (2013) 52. doi:10.5395/rde.2013.38.1.52.

[42] K.D. Hopkins, D.L. Weeks, Tests for Normality and Measures of Skewness and Kurtosis: Their Place in Research Reporting, Educ. Psychol. Meas. 50 (1990) 717-729. doi:10.1177/0013164490504001.

[43] J.M. Hedgepeth, P. Van Dyke, Local Stress Concentrations in Imperfect Filamentary Composite Materials, J. Compos. Mater. 1 (1967) 294-309. doi:10.1177/002199836700100305.

[44] C.M. Landis, R.M. McMeeking, Stress concentrations in composites with interface sliding, matrix stiffness and uneven fiber spacing using shear lag theory, Int. J. Solids Struct. 36 (1999) 4333-4361. doi:10.1016/S0020-7683(98)00193-0.

[45] W.A. Curtin, N. Takeda, Tensile Strength of Fiber-Reinforced Composites: I. Model and Effects of Local Fiber Geometry, J. Compos. Mater. 32 (1998) 2042-2059. doi:10.1177/002199839803202203.

[46] Y. Swolfs, L. Gorbatikh, V. Romanov, S. Orlova, S. V. Lomov, I. Verpoest, Stress concentrations in an impregnated fibre bundle with random fibre packing, Compos. Sci. Technol. 74 (2013) 113-120. doi:10.1016/j.compscitech.2012.10.013.

[47] D.G. Harlow, S.L. Phoenix, Probability distributions for the strength of composite materials II: A convergent sequence of tight bounds, Int. J. Fract. 17 (1981) 601-630. doi:10.1007/BF00681559.

[48] W.A. Curtin, Tensile Strength of Fiber-Reinforced Composites: III. Beyond the Traditional Weibull Model for Fiber Strengths, J. Compos. Mater. 34 (2000) 1301-1332. doi:10.1106/0gu5-Imta-9r99-2w8k. 